\author{
Series A
}

I. MATHEMATICA

448

\title{
DEFINITIONS FOR QUASIREGULAR MAPPINGS
}

BY

O. MARTIO, S. RICKMAN and J. VÄISÄLÄ

H E L S I N K I 1969

S U O M A L A I N E N T I EDE A K A T E M I A

doi:10.5186/aasfm.1969.448 
Communicated 9 May 1969 by Olli Lehto and K. I. Virtanen 


\section{Contents}

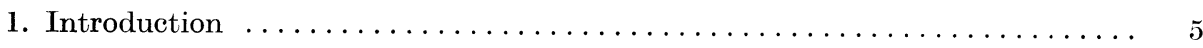

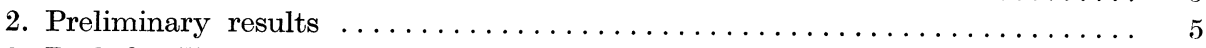

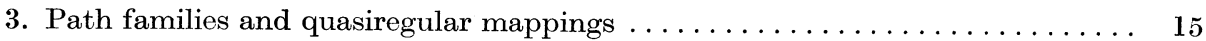

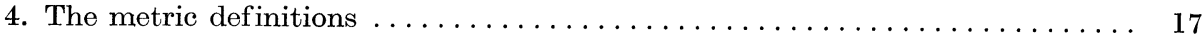

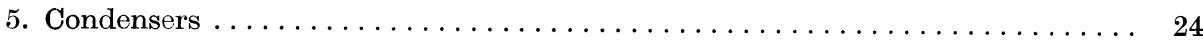

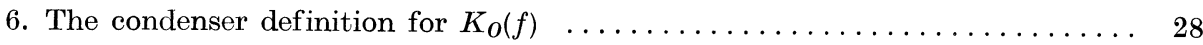

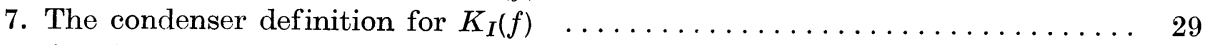

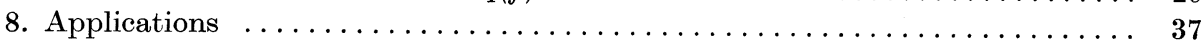

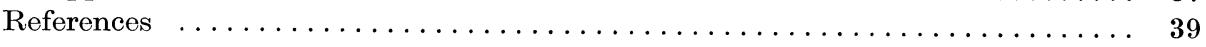




\section{Introduction}

The theory of quasiconformal mappings in the euclidean $n$-space has been quite extensively studied during the last ten years. Usually, a quasiconformal mapping is assumed to be a homeomorphism. For $n=2$, there exists also a theory of non-homeomorphic quasiconformal mappings. See Lehto-Virtanen [13, Kapitel VI] and Künzi [12, Kapitel 5]. These mappings are often called quasiconformal functions (not mappings). Some authors call them pseudoanalytic functions, but this term has been used by Bers in a different sense. We prefer the word quasiregular, and do not make any distinction between the words "mapping» and "function». It is fairly easy to generalize several function-theoretic results for 2-dimensional quasiregular mappings, thanks to the following factorization theorem: Every 2-dimensional quasiregular mapping can be represented in the form $g \circ h$ where $h$ is a quasiconformal homeomorphism and $g$ is a complex analytic function.

Higher dimensional quasiregular mappings, under the name "mappings with bounded distortion", have been considered by Rešetnjak since 1966 in a series of important papers $[15,16,17,18,19,20]$. (See also Callender [2].) He uses an analytic definition which will be given in 2.20. He also hints at a geometric definition in $[16$, p. 629]. The purpose of this paper is to give several equivalent characterizations for quasiregularity. These are based on the linear dilatations and on the capacity of a condenser. The last concept, defined in Section 5, is a generalization of the modulus of a ring domain. Some of these results are new also for $n=2$. In Section 8 , we give some applications. For example, we show that the branch set of a nonconstant quasiregular mapping has measure zero.

\section{Preliminary results}

2.1. Notation and terminology. The real number system is denoted by $R^{1}$ and its two-point compactification $R^{1} \cup\{\infty,-\infty\}$ by $\dot{R}^{1}$. We let $R^{n}, n \geqq 2$, denote the euclidean $n$-space, and $R^{n-1}$ will be identified with the subspace $x_{n}=0$ of $R^{n}$. For $x \in R^{n}$ we write $x=x_{1} e_{1}+\cdots+x_{n} e_{n}$ where $e_{1}, \ldots, e_{n}$ are the coordinate unit vectors of $R^{n}$. For each set 
$A \subset R^{n}$ we let $\mathbf{C} A, \bar{A}, \partial A$, and $\operatorname{int} A$ denote the complement, closure, boundary, and interior of $A$, all taken with respect to $R^{n}$. Furthermore, $d(A)$ is the diameter of $A$. Given two sets $A$ and $B$ in $R^{n}, d(A, B)$ is the distance between $A$ and $B, A \backslash B$ is the set-theoretic difference of $A$ and $B$, and $A+B$ is the set of all points $a+b$ such that $a \in A$ and $b \in B$. Given $x \in R^{n}$ and $r>0$, we let $B^{n}(x, r)$ denote the open ball $\left\{y \in R^{n}|| y-x \mid<r\right\}$, and $S^{n-1}(x, r)$ the sphere $\partial B^{n}(x, r)$. We shall also employ the abbreviations

$B^{n}(r)=B^{n}(0, r), B^{n}=B^{n}(1), S^{n-1}(r)=S^{n-1}(0, r), S^{n-1}=S^{n-1}(1)$.

The Lebesgue measure of a set $A \subset R^{n}$ will be written as $m_{n}(A)$, or simply as $m(A)$ if there is no danger of misunderstanding. $m_{n}(A)$ is also defined for sets in $n$-dimensional spheres and linear submanifolds of $R^{n^{\prime}}, n^{\prime}>n$. The Lebesgue integral of a function $f$ over a set $A \subset R^{n}$ is written as

$$
\int_{\boldsymbol{A}} f d m_{n} \text { or } \int_{\boldsymbol{A}} f(x) d m_{n}(x),
$$

where the subscript $n$ may again be omitted. We set $\Omega_{n}=m_{n}\left(B^{n}\right)$ and $\omega_{n-1}=n \Omega_{n}=m_{n-1}\left(S^{n-1}\right)$. The linear measure $\Lambda_{1}(A)$ of a set $A \subset R^{n}$ is defined as follows: For $t>0$ let

$$
A_{1}^{t}(A)=\inf \sum_{i=1}^{\infty} d\left(A_{i}\right)
$$

over all countable coverings $\left\{A_{1}, A_{2}, \ldots\right\}$ of $A$ such that $d\left(A_{i}\right)<t$. Then

$$
\Lambda_{1}(A)=\lim _{t \rightarrow 0} \Lambda_{1}^{t}(A)=\sup _{t>0} \Lambda_{1}^{t}(A) .
$$

If $A \subset R^{n}$ is a Borel set, Bor $A$ denotes the class of all Borel subsets of $A$. A neighborhood of a point $x$ or a set $A$ is an open set containing $x$ or $A$. A domain is an open connected non-empty set. The notation $f: G \rightarrow R^{n}$ includes the assumptions that $G$ is a domain in $R^{n}$ and that $f$ is continuous. If $f: G \rightarrow R^{n}, A \subset G$ and $y \in R^{n}$, we let $N(y, f, A)$ be the number (possibly infinite) of points in $A \cap f^{-1}(y)$. We set $N(f, A)$ $=\sup N(y, f, A)$ over $y \in R^{n}$.

Given a domain $G \subset R^{n}$, we let $J(G)$ denote the family of all domains $D$ such that $\bar{D}$ is a compact subset of $G$. If $f: G \rightarrow R^{n}, D \in J(G)$, and $y \in \mathbf{C} f \partial D$, then $\mu(y, f, D)$ is the degree (topological index) of the triple $(y, f, D)[14, p .125]$. Suppose that $x \in G$ has a connected neighborhood $D \in J(G)$ such that $\bar{D} \cap f^{-1}(f(x))=\{x\}$. Then $\mu(f(x), f, D)$ is independent of the choice of $D$, and is denoted by $i(x, f)$. 
The $i^{\text {th }}$ partial derivative of a mapping $f: G \rightarrow R^{n}$ is denoted by $\partial_{i} f$. If all partial derivatives of $f$ exist at a point $x \in G$, the formal derivative of $f$ at $x$ is the linear mapping $f^{\prime}(x): R^{n} \rightarrow R^{n}$, defined by $f^{\prime}(x) e_{i}=\partial_{i} f(x), 1 \leqq i \leqq n$. If $f$ is differentiable at $x$, then $f^{\prime}(x)$ is the derivative of $f$, that is,

$$
f(x+h)=f(x)+f^{\prime}(x) h+|h| \varepsilon(x, h)
$$

where $\varepsilon(x, h) \rightarrow 0$ as $h \rightarrow 0$. The Jacobian of $f$ at $x$ ls denoted by $J(x, f)$.

If $T: R^{n} \rightarrow R^{n}$ is a linear mapping, we set

$$
|T|=\max _{|x|=1}|T x|, l(T)=\min _{|x|=1}|T x| .
$$

If $U$ is an open set in $R^{n}$, we let $C^{p}(U)$ denote the class of all $p$ times continuously differentiable functions $u: U \rightarrow R^{1}$, and $C_{0}^{p}(U)$ the class of all $u \in C^{p}(U)$ whose support spt $u$ is a compact subset of $U$. A function in $C_{0}^{p}(U)$ will be identified in a natural way with a function in $C_{0}^{p}\left(R^{n}\right)$, which vanishes in $\mathbf{c} U$.

Suppose that $f: G \rightarrow R^{n}$. The branch set $B_{f}$ of $f$ is the set of all points of $G$ at which $f$ fails to be a local homeomorphism. $f$ is open if the image of every open set in $G$ is open in $R^{n} . f$ is light if for every $y \in R^{n}$, $f^{-1}(y)$ is totally disconnected. $f$ is discrete if for every $y \in R^{n}, f^{-1}(y)$ is discrete, that is, consists of isolated points. $f$ is sense-preserving if $\mu(y, f, D)>0$ whenever $D \in J(G)$ and $y \in f D \backslash f \partial D . f$ is sense-reversing if $\mu(y, f, D)<0$ for all such triples $(y, f, D) \cdot f$ satisfies the condition $(N)$ if the image of every set of measure zero has measure zero.

A continuum is a compact connected non-empty set. A ring is a domain $A \subset R^{n}$ such that $\hat{K}^{n} \backslash A$ has exactly two components, where $\hat{K}^{n}$ is the one point compactification of $R^{n}$.

2.2. Quasiadditive set functions. Let $U$ be an open set in $R^{n}$. A mapping $\varphi:$ Bor $U \rightarrow \dot{R}^{1}$ is said to be a $q$-quasiadditive set function, $q \geqq 1$, if the following conditions are satisfied for all Borel sets in $U$ :

(1) $\varphi(A) \geqq 0$.

(2) $A \subset B$ implies $\varphi(A) \leqq \varphi(B)$.

(3) $\varphi(A)<\infty$ if $A$ is compact.

(4) If $A_{1}, \ldots, A_{k}$ are disjoint and if $A_{i} \subset A$, then

$$
\sum_{i=1}^{k} \varphi\left(A_{i}\right) \leqq q \varphi(A) .
$$

From (4) it follows that the same inequality is true for an infinite sequence of disjoint Borel sets $A_{1}, A_{2}, \ldots$. 
The upper and lower derivatives of a $q$-quasiadditive set function $p$ at a point $x \in U$ are defined as follows:

$$
\begin{aligned}
& \bar{\varphi}^{\prime}(x)=\lim _{h \rightarrow 0} \sup _{d(Q)<h} \frac{\varphi(Q)}{m(Q)}, \\
& \underline{\varphi}^{\prime}(x)=\lim _{h \rightarrow 0} \inf _{d(Q)<h} \frac{\varphi(Q)}{m(Q)},
\end{aligned}
$$

where $Q$ runs through all open cubes and open balls such that $x \in Q \subset U$.

2.3. Lemma. Suppose that $p$ is a q-quasiadditive set function in an open set $U$. Then

(1) $\bar{\varphi}^{\prime}$ and $\varphi^{\prime}$ are Borel functions.

(2) $\bar{\varphi}^{\prime}(x) \leqq \bar{q} \varphi^{\prime}(x)<\infty$ a.e.

(3) For each open set $V \subset U$,

$$
\int_{V} \underline{\varphi}^{\prime} d m \leqq q \varphi(V) .
$$

The proof for $q=1$ is given in [14, pp. 204-209]. The proof for the general case is completely analogous. The definition of [14] for the derivatives $\bar{\varphi}^{\prime}, \varphi^{\prime}$ is slightly different from ours, because the sets $Q$ are in [14] assumed to be cubes. However, this makes no difference in the proof of 2.3 , since the Vitali covering theorem holds for the family of all closed cubes and closed balls.

2.4. Normal domains. Given a mapping $f: G \rightarrow R^{n}$, a domain $D \in J(G)$ is said to be a normal domain of $f$ if $f \partial D=\partial f D$. A normal neighborhood of a point $x \in G$ is a normal domain $D$ such that $D \cap f^{-1}(f(x))=\{x\}$.

We shall use the concept of a normal domain only for open mappings. In this case, we have always $\partial f D \subset f \partial D$, and the condition $f \partial D \subset \partial f D$ means that $f$ defines a closed mapping $D \rightarrow f D$. The definition of Whyburn [28] for a normal domain is slightly more restrictive since he also demands thas $f$ defines an open mapping $\bar{D} \rightarrow f \bar{D}$. It is not difficult to show that, for discrete open mappings, the domains $U(x, f, r)$, which will be defined in 2.8 and used throughout the paper, satisfy this additional condition, but we shall not use this fact.

If $D$ is a normal domain of an open mapping $f$, then $f D \cap f \partial D=\varnothing$. Hence, $\mu(y, f, D)$ is constant for $y \in f D$. This constant will be denoted by $\mu(f, D)$. 
2.5. Lemma. Suppose that $f: G \rightarrow R^{n}$ is open, that $U \subset R^{n}$ is a domain, and that $D$ is a component of $f^{-1} U$ such that $D \in J(G)$. Then $D$ is a normal domain, $f D=U$, and $U \in J(f G)$.

Proof. Since $f$ is open, $\partial f D \subset f \partial D$. Next assume $y \in f \partial D$. Then $y=f(x)$ for some $x \in \partial D$. Now $x \notin f^{-1} U$, since otherwise $D$ would not be a component of $f^{-1} U$. Thus, $y \notin U \supset f D$. Hence $y \in f \bar{D} \backslash f D=$ $\overline{f D} \backslash f D=\partial f D$. Consequently, $D$ is a normal domain. Furthermore, $f \partial D \cap U=\varnothing$. Thus $f D=U \cap f \bar{D}$ is both closed and open in $U$, whence $f D=U$. Finally, $\bar{U}=f \bar{D}$ is a compact subset of $f G$, i.e., $U \in J(f G)$.

2.6. Lemma. Suppose that $f: G \rightarrow R^{n}$ is open and that $D$ is a normal domain of $f$. If $E$ is either a domain or a continuum in $f D$, then $f$ maps every component of $D \cap f^{-1} E$ onto $E$. Furthermore, if $F$ is a compact subset of $f D$, then $D \cap f^{-1} F$ is compact.

Proof. The case where $E$ is a domain follows from 2.5. If $E \subset f D$ is compact, then $D \cap f^{-1} E=\bar{D} \cap f^{-1} E$ is compact. Moreover, $f$ defines an open mapping $D \cap f^{-1} E \rightarrow E$ ([27, (7.2), p. 147]). If $E$ is a continuum, every component of $D \cap f^{-1} E$ is mapped onto $E$ by [27, (7.5), p. 148].

2.7 Lemma. (Path lifting). Suppose that $f: G \rightarrow R^{n}$ is light and open and that $D \subset G$ is a normal domain. Suppose also that $\beta:[a, b] \rightarrow f D$ is a path, that $a \leqq t_{0} \leqq b$, and that $x_{0} \in D$ such that $f\left(x_{0}\right)=\beta\left(t_{0}\right)$. Then there is a path $\alpha:[a, b] \rightarrow D$ such that $\left.\alpha^{(} t_{n}\right)=x_{0}$ and $f \circ \alpha=\beta$.

Proof. Considering the restrictions of $\beta$ to $\left[a, t_{0}\right]$ and $\left[t_{0}, b\right]$ separately, we may assume that $t_{0}$ is an end point, say $t_{0}=a$. Set $I=[a, b]$, $J^{\prime}=\beta I$, and $J=D \cap f^{-1} J^{\prime}$. Then $J$ and $J^{\prime}$ are compact, and $f$ defines an open mapping $J \rightarrow J^{\prime}$. Define $g: J \times I \rightarrow J^{\prime} \times I$ by $g(x, t)$ $=(f(x), t)$ and $\beta_{1}: I \rightarrow J^{\prime} \times I$ by $\beta_{1}(t)=(\beta(t), t)$. Then $g$ is a light open mapping, and $\beta_{1} I$ is an arc. By a result of Whyburn [27, (2.1), p. 186] there is an arc $J_{0} \subset J \times I$ such that $\left(x_{0}, a\right) \in J$ and such that $g$ maps $J_{0}$ homeomorphically onto $\beta_{1} I$. Set $\alpha=P \circ\left(g \mid J_{0}\right)^{-1} \circ \beta_{1}$ where $P: J \times I \rightarrow J$ is the projection. Then $\alpha\left(t_{0}\right)=x_{0}$ and $f \circ \alpha=\beta$.

2.8. Notation. If $f: G \rightarrow R^{n}, x \in G$, and $r>0$, then the $x$-component of $f^{-1} B^{n}(f(x), r)$ is denoted by $U(x, f, r)$.

2.9. Lemma. Suppose that $f: G \rightarrow R^{n}$ is discrete and open. Then $\lim _{r \rightarrow 0} d(U(x, f, r))=0$ for every $x \in G$. If $U(x, f, r) \in J(G)$, then $U(x, f, r)$ 
is a normal domain and $f U(x, f, r)=B^{n}(f(x), r) \in J(f(G))$. Furthermore, for every point $x \in G$ there is a positive number $\sigma_{x}$ such that the following conditions are satisifed for $0<r \leqq \sigma_{x}$ :

(1) $U(x, f, r)$ is a normal neighbourhood of $x$.

(2) $U(x, f, r)=U\left(x, f, \sigma_{x}\right) \cap f^{-1} B^{n}(f(x), r)$.

(3) $\partial U(x, f, r)=U\left(x, f, \sigma_{x}\right) \cap f^{-1} S^{n-1}(f(x), r)$ if $r<\sigma_{x}$.

(4) $\mathbf{C} U(x, f, r)$ is connected.

(5) $\mathbf{c} \bar{U}(x, f, r)$ is connected.

(6) If $0<r<s \leqq \sigma_{x}$, then $\bar{U}(x, f, r) \subset U(x, f, s)$, and $U(x, f, s)$ $\backslash \bar{U}(x, f, r)$ is a ring.

Proof. Given $x \in G$ and $\varepsilon>0$, choose a neighborhood $W$ of $x$ such that $W \in J(G), d(W)<\varepsilon$, and $\bar{W} \cap f^{-1}(f(x))=\{x\}$. Then $U(x, f, r) \subset W$ for $0<r<d(f(x), f \partial W)$. Hence $d(U(x, f, r)) \rightarrow 0$ as $r \rightarrow 0$. If $U(x, f, r) \in J(G)$, it follows from 2.5 that $U(x, f, r)$ is a normal domain and that $f U(x, f, r)=B^{n}(f(x), r) \in J(f G)$.

The first part of the proof implies that $x$ has a normal neighborhood $D$. We choose $\sigma_{x}$ such that $0<\sigma_{x}<d(f(x), f \partial D)$ and such that $U\left(x, f, \sigma_{x}\right)$ $\subset B^{n}(x, t) \subset D$ for some $t>0$. To verify the properties $(1)-(6)$ we may assume that $0<r<\sigma_{x}$. Set $U=U(x, f, r), U_{0}=U\left(x, f, \sigma_{x}\right)$, and $V=f U=B^{n}(f(x), r)$. The condition (1) is clear by what was proved above. Since $U_{0} \cap f^{-1}(f(x))=\{x\}, \quad$ (2) follows from 2.6. Suppose next that $z \in U_{0} \cap f^{-1} \partial V$. Since $f$ is open, every neighborhood of $z$ meets $f^{-1} V$. By (2), this implies $z \in \bar{U}$. Thus $z \in \partial U$, whence $U_{0} \cap f^{-1} \partial V \subset \partial U$. On the other hand, $f \partial U=\partial f U=\partial V$ implies $\partial U \subset U_{0} \cap f^{-1} \partial V$, and (3) is proved.

Since $U \subset B^{n}(x, t) \subset D$, there is exactly one component $E$ of $\mathbf{C} U$ which meets $\mathbf{C} D$. We show that $E=\mathbf{C} U$. Set $F=\mathbf{C} U \backslash E$. Since $D \cap f^{-1}(f(x))=\{x\}$, it follows from 2.6 that $U=D \cap f^{-1} V$. Hence $f F$ does not meet $V$. Since $f$ is open, $\partial f F \subset f \partial F \subset f \partial U=\partial V$. Since $f F$ is bounded, $f F \subset \partial V$. Since $f$ is open, $\operatorname{int} F=\varnothing$. Setting $U_{1}=\mathbf{C} E$ we thus have $\bar{U}_{1}=\bar{U}$. Hence $f U_{1} \subset \operatorname{int} f \bar{U}=V$, which implies $f F \subset V$. This proves $F=\varnothing$. Thus, $\mathbf{C} U=E$ is connected.

If $x, y \in \mathbf{C} \bar{U}$, there is $r_{1}$ such that $r<r_{1}<\sigma_{x}$ and such that $x, y \in$ $\mathbf{C} U\left(x, f, r_{1}\right) \subset \mathbf{C} \bar{U}$. Hence (5) follows from (4).

The relation $\bar{U}(x, f, r) \subset U(x, f, s)$ follows from the last statement of 2.6. By (4), the components of the complement of $A=U(x, f, s) \backslash$ $\bar{U}(x, f, r)$ are $\bar{U}(x, f, r)$ and $\mathbf{C} U(x, f, s)$. By (5) and the PhragménBrouwer theorem [11, p. 359], $A$ is connected. Thus $A$ is a ring.

2.10. Corollary. If $f: G \rightarrow R^{n}$ is discrete and open, then every point in $G$ has arbitrarily small normal neighborhoods. 
2.11. Lemma. If $f: G \rightarrow R^{n}$ is discrete and open, $\operatorname{dim} B_{f} \leqq n-2$.

This important result was proved by Černavskii $[3,4]$. Another proof is given in [25]. It implies that $G \backslash B_{f}$ is connected. Hence $i(x, f)$ has a constant value, either +1 or -1 , in $G \backslash B_{f}$. In the first case $f$ is sense-preserving, and in the second case sense-reversing. For convenience, we shall restrict ourselves in this paper to sense-preserving mappings. This is obviously an unessential restriction.

2.12. Lemma. Suppose that $f: G \rightarrow R^{n}$ is sense-preserving, discrete, and open.

(1) If $D \in J(G)$, then $N(y, f, D) \leqq \mu(y, f, D)$ for all $y \in \mathbf{C} f \partial D$, and $N(y, f, D)=\mu(y, f, D)$ for $y \in \mathbf{C} f\left(\partial D \cup\left(D \cap B_{f}\right)\right)$.

(2) If $D$ is a normal domain, $N(f, D)=\mu(f, D)$.

(3) If $A \subset G$ is compact, $N(f, A)<\infty$.

(4) Every point $x \in G$ has a neighborhood $V$ such that if $U$ is a neighborhood of $x$ and if $U \subset V$, then $N(f, U)=i(x, f)$.

(5) $x \in B_{f}$ if and only if $i(x, f) \geqq 2$.

Proof. (1) Let $y \in \mathbf{C} f \partial D$ and let $D \cap f^{-1}(y)=\left\{x_{1}, \ldots, x_{k}\right\}$. Then

$$
\mu(y, f, D)=\sum_{j=1}^{k} i\left(x_{j}, f\right) .
$$

Since $f$ is sense-preserving, $\quad i\left(x_{j}, f\right) \geqq 1$. Thus $\mu(y, f, D) \geqq k=$ $N(y, f, D)$. If $y \in \mathbf{C} f\left(\partial D \cup\left(D \cap B_{f}\right)\right)$, every $i\left(x_{j}, f\right)=1$ in the above sum, and we have $\mu(y, f, D)=N(y, f, D)$.

(2) By [5, 2.2], $\operatorname{dim} f B_{f} \leqq n-2<n$. Hence there is a point $y \in f D \backslash$ $f B_{f}$. By (1), $\mu(f, D)=\mu(y, f, D)=N(y, f, D) \leqq N(f, D)$. On the other hand, (1) implies that $N(z, f, D) \leqq \mu(f, D)$ for all $z \in \mathbf{C} f \partial D$. Hence, $\mu(f, D)=N(f, D)$.

(3) By 2.9, $A$ can be covered by a finite number of normal domains $D_{1}, \ldots, D_{k}$. Using (2) we obtain

$$
N(f, A) \leqq \sum_{i=1}^{k} N\left(f, D_{i}\right)=\sum_{i=1}^{k} \mu\left(f, D_{i}\right)<\infty .
$$

(4) By 2.9, $x$ has a normal neighborhood $V$. If $U \subset V$ is a neighborhood of $x$, there is a normal neighborhood $V_{1}$ of $x$ such that $V_{1} \subset U$. Then (2) implies $i(x, f)=N\left(f, V_{1}\right) \leqq N(f, U) \leqq N(f, V)=i(x, f)$.

(5) follows from (4).

2.13. Remark. Since a light sense-preserving mapping is discrete and open [22, p. 333], we could replace the words "sense-preserving, discrete, 
and open» by "sense-preserving and light» throughout the paper. However, we shall not do this, because it is essential that our mappings are discrete and open, while sense-preservation is assumed mainly for the sake of convenience.

2.14. Lemma. Suppose that $f: G \rightarrow R^{n}$ is sense-preserving, discrete, and open, and that $f$ is differentiable at $x_{0} \in G$. Then $J\left(x_{0}, f\right) \geqq 0$. If $x_{0} \in B_{f}, J\left(x_{0}, f\right)=0$. If $A$ is a Borel set in $G$ and if $f$ is differentiable a.e. in $A$, then

$$
\int_{A} J(x, f) d m(x) \leqq \int_{R^{n}} N(y, f, A) d m(y) .
$$

Proof. If $J\left(x_{0}, f\right) \neq 0$, then $i\left(x_{0}, f\right)=\operatorname{sgn} J\left(x_{0}, f\right)$ by $[14,(68)$, p. 332]. Since $f$ is sense-preserving, $J\left(x_{0}, f\right) \geqq 0$. By 2.12, $i(x, f) \geqq 2$ for $x \in B_{f}$. Thus $J\left(x_{0}, f\right)=0$ if $x_{0} \in B_{f}$. The inequality (2.15) can be derived from general integral inequalities (see [14, p. 260]), but it can also be proved directly as follows. We express $A \backslash B_{f}$ as a union of disjoint Borel sets $A_{1}, A_{2}, \ldots$ such that each $A_{i}$ is contained in a domain $D_{i} \subset D$ in which $f$ is injective. Since (2.15) is well known to be true for homeomorphisms and since $J(x, f)=0$ a.e. in $A \cap B_{f}$, we obtain

$$
\begin{gathered}
\int_{A} J(x, f) d m(x)=\int_{A \backslash B_{f}} J(x, f) d m(x)=\sum \int_{A_{i}} J(x, f) d m(x) \\
\leqq \sum \int_{R^{n}} N\left(y, f, A_{i}\right) d m(y)=\int_{R^{n}} \sum N\left(y, f, A_{i}\right) d m(y) \leqq \int_{R^{n}} N(y, f, A) d m(y) .
\end{gathered}
$$

2.16. ACL-mappings. Let $R_{i}^{n-1}$ be the subspace of $R^{n}$ defined by $x_{i}=0$, and let $P_{i}: R^{n} \rightarrow R_{i}^{n-1}$ be the orthogonal projection. Suppose that $U$ is an open set in $R^{n}$. A mapping $g: U \rightarrow R^{m}$ is said to be ACL if $g$ is continuous and if for each open $n$-interval $Q$ such that $\bar{Q} \subset U$. $g$ is absolutely continuous on almost every line segment in $\bar{Q}$, parallel to the coordinate axes. More precisely, if $E_{i}$ is the set of all points $\tilde{z} \in P_{i} Q$ such that $g$ is not absolutely continuous on $\bar{Q} \cap P_{i}^{-1}(z)$, then $m_{n-1}\left(E_{i}\right)$ $=0,1 \leqq i \leqq n$. An ACL-mapping has partial derivatives a.e. If these are locally $L^{p}$-integrable, $p \geqq 1, g$ is said to be $\mathrm{ACL}^{p}$.

Suppose that $f: G \rightarrow R^{n}$ is a discrete open mapping and that $Q \in J(G)$ is an $n$-interval. For each Borel set $A \subset P_{i} Q$ we set $\varphi_{i}(A, Q)=$ $m\left(f\left(Q \cap P_{i}^{-1} A\right)\right)$. Then $A \mapsto \varphi_{i}(A, Q)$ is a $q$-quasiadditive set function, where $q=N(f, Q)<\infty$ by 2.12. Hence, by 2.3, its upper derivative $\bar{\varphi}_{i}^{\prime}(z, Q)$ is finite for almost every $z \in P_{i} Q$. 
2.17. Lemma. Suppose that $f: G \rightarrow R^{n}$ is discrete and open and that for every domain $D \in J(G)$ there is a finite constant $C_{D}$ such that

$$
\left(\sum_{j=1}^{k} d\left(f \Delta_{J}\right)\right)^{n} \leqq C_{D} \bar{\varphi}_{i}^{\prime}(z, Q)\left(\sum_{j=1}^{k} m_{1}\left(\Delta_{j}\right)\right)^{n-1}
$$

whenever $Q$ is an open n-interval in $D, 1 \leqq i \leqq n, z \in P_{i} Q$, and $\Delta_{1}, \ldots$, $\Delta_{k}$ are disjoint closed subintervals of $Q \cap P_{i}^{-1}(z)$. Then $f$ is $\mathrm{ACL}^{n}$.

Proof. This lemma is a generalization of a result of Agard [1]. The following proof is essentially due to him.

Let $Q \in J(G)$ be an open $n$-interval. A simple limiting process shows that $(2.18)$ is true whenever $\Delta_{1}, \ldots, \Delta_{k}$ are non-overlapping subintervals of $\bar{Q} \cap P_{i}^{-1}(z)$. Thus $f$ is ACL. To prove that $f$ is $\mathrm{ACL}^{n}$, it suffices to show, by symmetry, that $\left|\partial_{n} f\right|^{n}$ is integrable over $Q$.

Choose an integer $j_{0}$ such that $0<1 / j_{0}<d(Q, \partial G)$ and set

$$
g(x)=\left|\partial_{n} f(x)\right|, g_{j}(x)=\frac{j}{2} \int_{-1 / j}^{1 / j} g\left(x+t e_{n}\right) d t .
$$

Then $g_{j}(x)$ is defined for almost every $x \in Q$ and for all $j \geqq j_{0}$. We first show that $g_{j} \rightarrow g$ a.e. in $Q$. It is well known that $g$ is measurable, in fact, $g$ is a Borel function (Saks [21, p. 170]). Hence, the function $(x, t) \mapsto g\left(x+t e_{n}\right)$ is measurable in $Q \times(-1 / j, 1 / j)$. By Fubini's theorem, this implies that $g_{j}$ is measurable. Write $Q=Q_{0} \times J$, where $Q_{0}=P_{n} Q$ is an open $(n-1)$-interval and $J=(a, b)$ is an open 1-interval. Then almost every $z \in Q_{0}$ has the property that $t \mapsto f(z, t)$ is absolutely continuous for $t \in\left(a-1 / j_{0}, b+1 / j_{0}\right)$. For such $z$, Lebesgue's theorem implies that $g_{j}(z, t) \rightarrow g(z, t)$ for almost every $t \in J$. From Fubini's theorem it follows that $\lim \inf g_{j}(x)=g(x)=\lim \sup g_{j}(x)$ a.e. in $Q$. Thus $g_{j} \rightarrow g$ a.e. in $Q$.

Again by Fubini's theorem, almost every $u \in J$ has the property that $g_{j}(z, u) \rightarrow g(z, u)$ for almost every $z \in Q_{0}$. Consider such $u$, and set

$$
F_{j}(E)=\varphi_{n}\left(E, Q_{0} \times(u-1 / j, u+1 / j)\right)
$$

for all Borel sets $E \subset Q_{0}$ and for $j \geqq j_{0}$. Then the set functions $F_{j}$ are $q$-quasiadditive for $q=N(f, D)$ where $D=Q_{0} \times\left(a-1 / j_{0}, b+1 / j_{0}\right)$. If $\bar{F}_{j}^{\prime}(z)<\infty$, it follows from (2.18) that the function $t \mapsto f(z, t)$ is absolutely continuous on $[u-1 / j, u+1 / j]$ and that its total variation is not greater than $\left(C_{D} \bar{F}_{j}^{\prime}(z)(2 / j)^{n-1}\right)^{1 / n}$. Consequently,

$$
g_{j}(z, u)^{n}=\left(\frac{j}{2} \int_{u-1 / j}^{u+1 / j}\left|\partial_{n} f(z, t)\right| d t\right)^{n} \leqq C_{D} \bar{F}_{j}^{\prime}(z) j / 2 .
$$


Integrating over $z \in Q_{0}$ and using 2.3 we obtain

$\int_{Q_{0}} g_{j}(z, u)^{n} d m_{n-1}(z) \leqq \frac{1}{2} C_{D} q^{2} j F_{j}\left(Q_{0}\right)=\frac{1}{2} C_{D} q^{2} j m\left(f\left(Q_{0} \times(u-1 / j, u+1 / j)\right)\right)$.

For each Borel set $E \subset J$ set $\psi(E)=m\left(f\left(Q_{0} \times E\right)\right)$. Then $\psi$ is a $q$-quasiadditive set function in $J$. As $j \rightarrow \infty$, (2.19) implies by Fatou's lemma

$$
\int_{Q_{0}} g(z, u)^{n} d m_{n-1}(z) \leqq C_{D} q^{2} \bar{\psi}^{\prime}(u)
$$

Integrating over $u \in J$ and using 2.3 we obtain

$$
\int_{Q} g^{n} d m \leqq C_{D} q^{4} \psi(J)=C_{D} q^{4} m(f Q)<\infty \text {. }
$$

Thus $g^{n}$ is integrable over $Q$.

2.20. Quasiregular mappings. A mapping $f: G \rightarrow R^{n}$ is said to be quasiregular if $f$ is $\mathrm{ACL}^{n}$ and if there exists a constant $K \geqq 1$ such that

$$
\left|f^{\prime}(x)\right|^{n} \leqq K J(x, f)
$$

a.e. in $G$. The smallest $K \geqq 1$ for which this inequality is true is called the outer dilatation of $f$ and denoted by $K_{o}(f)$. If $f$ is quasiregular, then the smallest $K \geqq 1$ for which the inequality

$$
J(x, f) \leqq K l\left(f^{\prime}(x)\right)^{n}
$$

holds a.e. in $G$ is called the inner dilatation of $f$ and denoted by $K_{I}(f)$. The maximal dilatation of $f$ is the number $K(f)=\max \left(K_{I}(f), K_{o}(f)\right)$. If $K(f) \leqq K, f$ is said to be $K$-quasiregular. If $f$ is not quasiregular, we set $K_{o}(f)=K_{I}(f)=K(f)=\infty$.

The above definition is a natural generalization of the analytic definition for quasiconformal mappings [24]. A sense-preserving mapping is $K$-quasiconformal if and only if it is a $K$-quasiregular homeomorphism.

It is not true that every $\mathrm{ACL}^{n}$-mapping which satisfies (2.22) is quasiregular. For example, the projection $f(x)=x_{1} e_{1}$ satisfies $(2.22)$ with $K=1$, since $J(x, f)=l\left(f^{\prime}(x)\right)=0$ everywhere.

The above definition has been used by Callender [2] and Rešetnjak [15] in a slightly different form. In their definition (2.21) is replaced by

$$
\left(\sum_{i=1}^{n} \sum_{j=1}^{n} \partial_{i} f_{j}(x)^{2}\right)^{n / 2} \leqq n^{n / 2} K J(x, f)
$$


where $K$ is sometimes replaced by $K^{n}$ or by $K^{n / 2}$. Let $K_{R}(f)$ be the smallest $K \geqq 1$ for which $(2.23)$ holds a.e. Then it is easy to show that

$$
K_{R}(f) \leqq K_{o}(f) \leqq n^{n / 2} K_{R}(f)
$$

and that these inequalities are the best possible. Furthermore, we have the inequalities

$$
K_{o}(f) \leqq K_{I}(f)^{n-1}, K_{I}(f) \leqq K_{o}(f)^{n-1},
$$

which also are the best possible.

2.26. Lemma. Let $f: G \rightarrow R^{n}$ be a quasiregular mapping. Then

(1) $f$ is either constant or sense-preserving, discrete, and open.

(2) $f$ is differentiable a.e.

(3) $f$ satisfies the condition $(N)$.

These important results are due to Rešetnjak $[16,18]$.

2.27. Lemma. Suppose that $f: G \rightarrow R^{n}$ is quasiregular. Then $f^{\prime}(x)=0$ a.e. in $B_{f}$. Moreover, $m\left(f B_{f}\right)=0$.

Proof. We may assume that $f$ is not constant. By 2.14, $J(x, f)=0$ a.e. in $B_{f}$. Hence $\left|f^{\prime}(x)\right|=0$ a.e. in $B_{f}$. From [14, Lemma 3, p. 360] it follows that

$$
\int_{R^{n}} N\left(y, f, B_{f}\right) d m(y)=\int_{B_{f}} J(x, f) d m(x)=0 .
$$

Hence $m\left(f B_{f}\right)=0$.

2.28. Lemma. Suppose that $f: G \rightarrow R^{n}$ is a non-constant quasiregular mapping and that every point in $G \backslash B_{f}$ has a neighborhood $U$ such that $K_{o}(f \mid U) \leqq a, K_{I}(f \mid U) \leqq b$. Then $K_{o}(f) \leqq a, K_{I}(f) \leqq b$.

Proof. By $2.27, f^{\prime}(x)=0$ a.e. in $B_{f}$. Hence, the inequalities (2.21) and (2.22) are automatically satisfied in $B_{f}$.

\section{Path families and quasiregular mappings}

3.1. Suppose that $A$ is a subset of $R^{n}$. By a path in $A$ we mean a continuous mapping $\gamma: \Delta \rightarrow A$ where $\Delta$ is a closed interval in $R^{1}$. If $\Gamma$ is a family of paths in $R^{n}$, we let $F(\Gamma)$ be the family of all non-negative Borel functions $\varrho: R^{n} \rightarrow \dot{R}^{1}$ such that 


$$
\int_{\gamma} \varrho d s \geqq 1
$$

for every rectifiable $\gamma \in \Gamma$. The modulus of $\Gamma$ is the number

$$
M(\Gamma)=\inf _{\varrho \in F(\Gamma)} \int_{R^{n}} \varrho^{n} d m .
$$

Suppose that $\Gamma$ is a family of paths in a domain $G$ and that $f: G \rightarrow R^{n}$ is a mapping. Then the family $f \Gamma$ of all paths $f \circ \gamma, \gamma \in \Gamma$, is called the image of $\Gamma$ under $f$. If $f: G \rightarrow G^{\prime}$ is a $K$-quasiconformal mapping, it is well known [23] that

$$
M(\Gamma) / K \leqq M(f \Gamma) \leqq K M(\Gamma)
$$

for every path family $\Gamma$ in $G$. We conjecture that the right hand inequality is true also for $K$-quasiregular mappings. As yet, we have been able to prove it only in special cases, for example, if $n=2$ or if $B_{f}=\varnothing$. The left hand inequality need not be true for non-homeomorphic quasiregular mappings, as is seen from the following counterexample: Let $\Gamma$ be the family of all horizontal segments which join the vertical lines $\operatorname{Re} z$ $=0$ and $\operatorname{Re} z=1$ in the complex plane $R^{2}$. If $f: R^{2} \rightarrow R^{2}$ is the analytic function $f(z)=e^{z}$, we have $M(\Gamma)=\infty, M(f \Gamma)=2 \pi$. However, we can establish the following inequality:

3.2. Theorem. Suppose that $f: G \rightarrow R^{n}$ is a quasiregular mapping and that $A$ is a Borel set in $G$ such that $N(f, A)<\infty$. If $\Gamma$ is a family of paths in $A$,

$$
M(\Gamma) \leqq N(f, A) K_{o}(f) M(f \Gamma)
$$

Proof. Set

$$
L(x, f)=\limsup _{h \rightarrow 0} \frac{|f(x+h)-f(x)|}{|h|}
$$

for $x \in G$. Thus $L(x, f)=\left|f^{\prime}(x)\right|$ whenever $f$ is differentiable at $x$. It is easy to see that $x \mapsto L(x, f)$ is a Borel function.

Suppose that $\varrho^{\prime} \in F(f \Gamma)$. Define $\varrho: R^{n} \rightarrow \dot{R}^{1}$ by setting

$$
\varrho(x)=\varrho^{\prime}(f(x)) L(x, f)
$$

for $x \in A$ and $\varrho(x)=0$ for $x \in \mathbf{C} A$. Let $\Gamma_{0}$ be the family of all rectifiable paths $\gamma \in \Gamma$ such that $f$ is absolutely continuous on $\gamma$. By this we mean that if $\gamma^{0}$ is the parametrization of $\gamma$ by means of its path length, 
then $f \circ \gamma^{0}$ is absolutely continuous. By a result of Fuglede ([6] or [23, p. 16]), $M\left(\Gamma_{0}\right)=M(\Gamma)$. From the formula concerning change of variables in integrals it follows that

$$
\int_{\gamma} \varrho d s \geqq \int_{f_{\circ \gamma}} \varrho^{\prime} d s \geqq 1
$$

for all $\gamma \in \Gamma_{0}$. Thus $\varrho \in F\left(\Gamma_{0}\right)$. A more detailed proof is given in [26]. Hence we obtain

$$
\begin{gathered}
M(\Gamma)=M\left(\Gamma_{0}\right) \leqq \int_{R^{n}} \varrho^{n} d m=\int_{A} \varrho^{\prime}(f(x))^{n} L(x, f)^{n} d m(x) \\
\leqq K_{0}(f) \int_{A} \varrho^{\prime}(f(x))^{n} J(x, f) d m(x) .
\end{gathered}
$$

Since $f$ is $\mathrm{ACL}^{n}, J(x, f)$ is integrable over every domain $D \in J(G)$. Thus, the transformation formula in [14, Theorem 3, p. 364] yields

$$
\begin{gathered}
\int_{A \cap D} \varrho^{\prime}(f(x))^{n} J(x, f) d m(x)=\int_{R^{n}} \varrho^{\prime}(y)^{n} N(y, f, A \cap D) d m(y) \\
\leqq N(f, A) \int_{R^{n}} \varrho^{\prime n} d m .
\end{gathered}
$$

The theorem cited above is formulated in [14] for finite-valued functions, but we may apply it to $\min \left(k, \varrho^{\prime n}\right)$ and let then $k \rightarrow \infty$. Since $D \in J(G)$ is arbitrary, we obtain

$$
M(\Gamma) \leqq N(f, A) K_{o}(f) \int_{R^{n}} \varrho^{\prime n} d m .
$$

Since this holds for every $\varrho^{\prime} \in F(f T)$, the theorem follows.

\section{The metric definitions}

4.1. Notation. Let $f: G \rightarrow R^{n}$ be discrete and open, and let $x \in G$. If $0<r<d(x, \partial G)$, we denote

$$
\begin{gathered}
l(x, f, r)=\inf _{|x-y|=r}|f(y)-f(x)|, \\
L(x, f, r)=\sup _{|x-y|=r}|f(y)-f(x)|=\sup _{|x-y| \leqq r}|f(y)-f(x)| .
\end{gathered}
$$


If $0<r<d(f(x), \partial f G)$, we denote

$$
\begin{gathered}
l^{*}(x, f, r)=\inf _{z \in \partial U(x, f, r)}|x-z|, \\
L^{*}(x, f, r)=\sup _{z \in \partial U(x, f, r)}|x-z|=\sup _{z \in \bar{U}(x, f, r)}|x-z| .
\end{gathered}
$$

Recall that $U(x, f, r)$ is the $x$-component of $f^{-1} B^{n}(f(x), r)$.

4.2. Definition. Let $f: G \rightarrow R^{n}$ be discrete and open. If $x \in G$, we call

$$
H(x, f)=\limsup _{r \rightarrow 0} \frac{L(x, f, r)}{l(x, f, r)}
$$

the linear dilatation of $f$ at $x$, and

$$
H^{*}(x, f)=\limsup _{r \rightarrow 0} \frac{L^{*}(x, f, r)}{l^{*}(x, f, r)}
$$

the inverse linear dilatation of $f$ at $x$.

In this section we first establish upper bounds for $H(x, f)$ and $H^{*}(x, f)$ when $f$ is a non-constant quasiregular mapping. The main results (Theorems 4.13 and 4.14) are that both these dilatations characterize non-constant quasiregular mappings. These characterizations are called the metric definitions for quasiregularity.

The inverse linear dilatation also plays an essential role in Section 7 . where the important inner dilatation inequality for the capacities of condensers is proved.

We assume now that in all the lemmas which appear in this section $f: G \rightarrow R^{n}$ is a discrete open mapping. Given three sets $A, B, C$ in $R^{n}$. a path $\gamma:[a, b] \rightarrow R^{n}$ is said to join $A$ and $B$ in $C$ if $\gamma(a) \in A$, $\gamma(b) \in B$ and $\gamma(t) \in C$ for $a<t<b$.

4.3. Lemma. Let $x \in G$ and let $\sigma_{x}$ be as in 2.9. Then $l^{*}(x, f, L(x$. $f, r))=L^{*}(x, f, l(x, f, r))=r$ for $0<r<l^{*}\left(x, f, \sigma_{x}\right)$.

Proof. Set $l=l(x, f, r)$ and $L=L(x, f, r)$. Obviously $l \leqq L<\sigma_{x}$. We prove $l^{*}(x, f, L)=r$. The proof for $L^{*}(x, f, l)=r$ is similar. Since $B^{n}(x, r) \subset U(x, f, L), l^{*}(x, f, L) \geqq r$. Choose $a \in S^{n-1}(x, r)$ such that $|f(a)-f(x)|=L . \quad$ By 2.9, $\partial U(x, f, L)=U\left(x, f, \sigma_{x}\right) \cap f^{-1} S^{n-1}(f(x), L)$. Thus $a \in \partial U(x, f, r)$, which implies $l^{*}(x, f, L) \leqq|a-x|=r$.

4.4 Lemma. Let $x \in G$ and let $\sigma_{x}$ be as in 2.9. For $0<s<t \leqq \sigma_{x}$ let $\Gamma(s, t)$ be the family of all paths which join $\partial U(x, f, s)$ and $\partial U(x, f, t)$ 
in $U(x, f, t) \backslash \bar{U}(x, f, s)$. Suppose that there exist constants $b$ and $\sigma$, $1 \leqq b<\infty, \quad 0<\sigma \leqq \sigma_{x}$, such that $M(\Gamma(s, t)) \leqq b M(f \Gamma(s, t))$ for all $0<s<t<\sigma$. Then $H(x, f) \leqq C<\infty$ where $C$ depends only on $n$ and $b$.

Proof. Assume $0<r<l^{*}\left(x, f, \sigma_{x}\right)$, and set $L=L(x, f, r), l=$ $l(x, f, r)$. Obviously $L<\sigma_{x}$. Suppose $l<L$. Then $f \Gamma(l, L)$ is a subfamily of the family of all paths joining $S^{n-1}(f(x), l)$ and $S^{n-1}(f(x), L)$ in $B^{n}(f(x), L) \backslash \bar{B}^{n}(f(x), l)$. Hence $M(f \Gamma(l, L)) \leqq \omega_{n-1} /(\log (L / l))^{n-1}[23$, p. 7]. By 4.3, $\partial U(x, f, l)$ and $\partial U(x, f, L)$ meet $S^{n-1}(x, r)$. From this and the fact that $U(x, f, L) \backslash \bar{U}(x, f, l)$ is by 2.9 a ring it follows the estimate $M(\Gamma(l, L)) \geqq a_{n}>0$ where $a_{n}$ depends only on $n[26,11.7]$. Since $M(\Gamma(l, L)) \leqq b M(f \Gamma(l, L))$, we obtain $L / l \leqq C$ where

$$
C=\exp \left(\left(\frac{b \omega_{n-1}}{a_{n}}\right)^{1 /(n-1)}\right)
$$

This proves the lemma.

The upper bound for $H(x, f)$ when $f$ is a non-constant quasiregular mapping follows now easily from the result of Section 3 and Lemma 4.4.

4.5. Theorem. Let $f: G \rightarrow R^{n}$ be a non-constant quasiregular mapping. Then for every $x \in G$

$$
H(x, f) \leqq C<\infty,
$$

where $C$ depends only on $n$ and the product $i(x, f) K_{o}(f)$.

Proof. Let $x \in G$. By 2.26, 2.12, and 3.2, the conditions in 4.4 are satisfied with $b=i(x, f) K_{o}(f) \geqq 1$ and some $\sigma>0$. The result follows from 4.4.

A similar result holds for $H^{*}(x, f)$ :

4.6. Theorem. Let $f: G \rightarrow R^{n}$ be a non-constant quasiregular mapping. Then for every $x \in G$

$$
H^{*}(x, f) \leqq H(x, f)^{2 i(x, f) K o(f)} \leqq C^{*}<\infty,
$$

where $C^{*}$ depends only on $n$ and the product $i(x, f) K_{o}(f)$.

Proof. Let $x \in G$. By 2.26, $f$ is sense-preserving, discrete, and open. Choose $\sigma_{x}$ as in 2.9, and set $D=U\left(x, f, \sigma_{x}\right)$. Let $t>0$ be such that $L(x, f, t)<\sigma_{x}$ and let $r_{0}>0$ be such that $U(x, f, r) \subset B^{n}(x, t)$ if $0<r \leqq r_{0}$. Assume $0<r \leqq r_{0}$, and set $L^{*}=L^{*}(x, f, r), l^{*}=$ $l^{*}(x, f, r), L=L\left(x, f, L^{*}\right)$, and $l=l\left(x, f, l^{*}\right)$. We choose a line $J$ 
through $f(x)$. Let $A^{\prime}=\{y|l<| y-f(x) \mid<L\}$, let $E^{\prime}$ and $F^{\prime}$ be the components of $J \cap \bar{A}^{\prime}$, and set $E=D \cap f^{-1} E^{\prime}, F=D \cap f^{-1} F^{\prime}$. If $E_{0}$ is any component of $E, f E_{0}=E^{\prime}$ by 2.6. By $2.9, \quad \partial U(x, f, l)=$ $D \cap f^{-1} S^{n-1}(f(x), l)$ and $\partial U(x, f, L)=D \cap f^{-1} S^{n-1}(f(x), L)$. Hence $E_{0}$ meets both $\partial U(x, f, l)$ and $\partial U(x, f, L)$. Since $\partial U(x, f, l) \subset$ $\bar{B}^{n}\left(x, l^{*}\right)$ and $\partial U(x, f, L) \subset \mathbf{C} B^{n}\left(x, L^{*}\right)$, we get thus $\varnothing \neq S^{n-1}(x, u) \cap$ $E_{0} \subset S^{n-1}(x, u) \cap E$, and similarly $S^{n-1}(x, u) \cap F \neq \varnothing$ for every $u, l^{*}<u<L^{*}$.

Set $A=\left\{z\left|l^{*}<\right| z-x \mid<L^{*}\right\}$, and let $\Gamma$ be the family of all paths which join $E$ and $F$ in $A$. Then $M(\Gamma) \geqq c_{n} \log \left(L^{*} / l^{*}\right)$ where $c_{n}>0$ is the $n$-modulus of the family of all paths joining $e_{n}$ and $-e_{n}$ in $S^{n-1}$ $[26,10.12]$. On the other hand, every path in $f \Gamma$ joins $E^{\prime}$ and $F^{\prime}$ in $A^{\prime}$, and hence $M(f \Gamma) \leqq c_{n} \log (L / l)$ by $[26,10.12]$. By 3.2 and 2.12 we get thus

$$
c_{n} \log \frac{L^{*}}{l^{*}} \leqq M(\Gamma) \leqq i(x, f) K_{o}(f) M(f \Gamma) \leqq i(x, f) K_{o}(f) c_{n} \log \left(\frac{L}{r} \frac{r}{l}\right) .
$$

But $r=l\left(x, f, L^{*}\right)=L\left(x, f, l^{*}\right)$, and letting $r \rightarrow 0$ we obtain

$$
H^{*}(x, f) \leqq H(x, f)^{2 i(x, f) K_{0}(f)} .
$$

Theorem 4.5 completes the proof.

4.7. Remark. Define a $K$-quasiregular mapping $g: R^{2} \rightarrow R^{2}$ by $g(z)=$ $(x+i K y)^{k}$ where we have used the complex notation $z=x+i y$, where $K>1$, and where $k$ is a positive integer. Then $H(0, g)=K^{k}$ and $i(0, g)=k$, which shows that the linear dilatation depends in general on the local degree.

We turn now to the converse problem and establish characterizations of a non-constant quasiregular mapping $f$ by $H(x, f)$ and $H^{*}(x, f)$. Recall that $f: G \rightarrow R^{n}$ is a discrete open mapping in the lemmas in this section.

4.8. Lemma. Let $C \subset G$ be compact. Then there exists $t>0$ such that the mapping $(x, s) \mapsto l^{*}(x, f, s)$ is continuous and the mapping $(x, s) \mapsto$ $L^{*}(x, f, s)$ is lower semi-continuous in the set $C \times(0, t)$.

Proof. For $x \in C$ let $s_{x}>0$ be such that $\bar{U}\left(x, f, s_{x}\right) \subset B^{n}(x, a)$ where $a=d(C, \partial G)$. We cover $C$ by sets $U\left(x_{i}, f, s_{x_{i}} / 2\right), i=1, \ldots$, $k$. Assume $x \in C$ and let $x \in U\left(x_{j}, f, s_{x_{j}} / 2\right)$. Then $U\left(x, f, s_{x_{j}} / 2\right) \subset$ $U\left(x_{j}, f, s_{x_{j}}\right) \quad$ and hence $\quad \bar{U}(x, f, s) \subset C+a B^{n} \quad$ if $\quad 0<s<t=$ $\min \left(s_{x_{1}} / 2, \ldots, s_{x_{k}} / 2\right)$. Assume $\left(x_{0}, s_{0}\right) \in C \times(0, t) \quad$ and $\quad 0<\varepsilon<$ $\min \left(l^{*}\left(x_{0}, f, s_{0}\right), d\left(U\left(x_{0}, f, s_{0}\right), \partial G\right)\right)$, and set $y_{0}=f\left(x_{0}\right)$. 
We show first that $(x, s) \mapsto l^{*}(x, f, s)$ is upper semicontinuous at $\left(x_{0}, s_{0}\right)$. Let $z \in \partial U\left(x_{0}, f, s_{0}\right)$ be such that $\left|x_{0}-z\right|=l^{*}\left(x_{0}, f, s_{0}\right)$. The set $f B^{n}(z, \varepsilon / 2)$ is a neighborhood of $f(z)$ and there is therefore a point $v \in B^{n}(z, \varepsilon / 2) \cap \mathbf{C} \bar{U}\left(x_{0}, f, s_{0}\right)$ such that $v^{\prime}=f(v) \notin \bar{B}^{n}\left(y_{0}, s_{0}\right)$. Set $\tau=$ $\left(\left|v^{\prime}-y_{0}\right|-s_{0}\right) / 2$ and let $\delta, 0<\delta<\varepsilon / 2$, be such that $\left|x-x_{0}\right|<\delta$ implies $\left|f(x)-y_{0}\right|<\tau$. If now $(x, s) \in C \times(0, t]$ such that $\left|x-x_{0}\right|<\delta$ and $\left|s-s_{0}\right|<\tau$, then $v^{\prime} \notin \bar{B}^{n}(f(x), s)$ and hence $v \notin \bar{U}(x, f, s)$. This implies $l^{*}(x, f, s)<|v-z|+\left|z-x_{0}\right|+\left|x_{0}-x\right|<l^{*}\left(x_{0}, f, s_{0}\right)+\varepsilon$.

To show that $(x, s) \mapsto l *(x, f, s)$ is lower semicontinuous at $\left(x_{0}, s_{0}\right)$, set $r=l^{*}\left(x_{0}, f, s_{0}\right)-\varepsilon / 2$. Then $\bar{B}^{n}\left(x_{0}, r\right) \subset U\left(x_{0}, f, s_{0}\right)$, and $2 \tau=$ $d\left(f \bar{B}^{n}\left(x_{0}, r\right), S^{n-1}\left(y_{0}, s_{0}\right)\right)$ is positive. Let $\delta, 0<\delta<\varepsilon / 2$, be such that $\left|x-x_{0}\right|<\delta$ implies $\left|f(x)-y_{0}\right|<\tau$. Assume $(x, s) \in C \times(0, t],\left|x-x_{0}\right|$ $<\delta$, and $\left|s-s_{0}\right|<\tau$. Then $f \bar{B}^{n}\left(x_{0}, r\right) \subset B^{n}(f(x), s)$ and hence $\bar{B}^{n}\left(x_{0}, r\right) \subset U(x, f, s)$ because $x \in \bar{B}^{n}\left(x_{0}, r\right)$. From this it follows that $l^{*}(x, f, s) \geqq r-\left|x-x_{0}\right| \geqq l^{*}\left(x_{0}, f, s_{0}\right)-\varepsilon$.

Finally, to prove the lower semicontinuity of $(x, s) \mapsto L^{*}(x, f, s)$ at $\left(x_{0}, s_{0}\right)$, let $z \in \partial U\left(x_{0}, f, s_{0}\right)$ be such that $\left|z-x_{0}\right|=L^{*}\left(x_{0}, f, s_{0}\right)$ and let $u \in U\left(x_{0}, f, s_{0}\right) \cap B^{n}(z, \varepsilon / 2)$. There exists a continuum $A$ in $U\left(x_{0}\right.$, $\left.f, s_{0}\right)$ such that $u \in A$ and $B^{n}\left(x_{0}, \varepsilon / 2\right) \subset A$. Then $f A$ is a compact set in $B^{n}\left(y_{0}, s_{0}\right)$ and $2 \tau=d\left(f A, S^{n-1}\left(y_{0}, s_{0}\right)\right)$ is positive. Again, let $\delta$, $0<\delta<\varepsilon / 2$, be such that $\left|x-x_{0}\right|<\delta$ implies $\left|f(x)-y_{0}\right|<\tau$. Assuming $(x, s) \in C \times(0, t], \quad\left|x-x_{0}\right|<\delta, \quad$ and $\left|s-s_{0}\right|<\tau$, we have $f A \subset$ $B^{n}(f(x), s)$ and therefore $A \subset U(x, f, s)$. Hence $L^{*}(x, f, s) \geqq|x-u| \geqq$ $L^{*}\left(x_{0}, f, s_{0}\right)-\varepsilon$.

4.9. Remark. Let $g: R^{3} \rightarrow R^{3}$ be the mapping defined in the cylindrical coordinates by $g(r, \varphi, z)=(r, 2 \varphi, z)$. Then $x \mapsto L^{*}(x, g, s)$, $s>0$, is discontinuous at points $x=(s, \varphi, z)$.

4.10. Lemma. Let $C \subset G$ be compact. Then there exists $\varrho>0$ such that for $0<r<\varrho$

(1) $x \mapsto l(x, f, r)$ and $x \mapsto L(x, f, r)$ are continuous in $C$,

(2) $x \mapsto l^{*}(x, f, L(x, f, r))$ is continuous in $C$,

(3) $x \mapsto L^{*}(x, f, L(x, f, r))$ is lower semicontinuous in $C$.

Proof. The condition (1) holds for $0<r<d(C, \partial G)$. Let $t$ be as in 4.8 and let $\varrho, 0<\varrho<d(C, \partial G)$, be such that $|f(y)-f(z)|<t$ whenever $|y-z|<\varrho, y \in C$, and $z \in G$. Then (2) and (3) follow from 4.8 and (1).

4.11. Lemma. If $H(x, f)$ or $H^{*}(x, f)$ is locally bounded, $f$ is $\mathrm{ACL}^{n}$. 
Proof. The proof is carried through in full detail for $H^{*}(x, f)$. By a simplified version one can prove the statement for $H(x, f)$. We shall show that the condition of Lemma 2.17 is satisfied. This is done by modifying the proof of Gehring [9, Lemma 9].

Let $D \in J(G)$, let $Q \subset D$ be an open $n$-interval, and let $P: R^{n} \rightarrow R^{n-1}$ be the orthogonal projection. For each Borel set $A \subset P Q$ we define $Z_{A}=$ $Q \cap P^{-1} A$. Setting $\varphi(A, Q)=m\left(f Z_{A}\right)$ we obtain a $q$-quasiadditive set function $A \mapsto \varphi(A, Q)$ in $P Q$, where $q=N(f, D)$. Fix $z \in P Q$ such that the upper derivative $\bar{\varphi}^{\prime}(z, Q)$ is finite, and set $J=Z_{z}$.

Let $F$ be a compact subset of $J$. There exists $\varrho>0$ such that (2) and (3) in 4.10 hold for $0<r<\varrho$ and $C=\bar{Q}$. Since $H^{*}(x, f)$ is locally bounded, there is a constant $c<\infty$ such that $H^{*}(x, f)<c$ for $x \in D$. Given an integer $k$ such that $0<1 / k<\min (d(F, \partial Q), \varrho)$ let $F_{k}$ be the set of all $x \in F$ such that $0<r<1 / k c$ implies $L^{*}(x, f, L(x, f, r)) / c$ $\leqq l^{*}(x, f, L(x, f, r))=r$. Then every $F_{k}$ is compact by 4.10 . Moreover, $F=\cup F_{k}$ by 4.3. Fix $k$, and choose $\varepsilon>0$ and $t>0$. By a well-known lemma $[7, \mathrm{p} .6]$ there is a $\delta, 0<\delta<1 / k c$, such that for every $r, 0<$ $r<\delta$, there exists a covering of $F_{k}$ by open segments $\Delta_{1}, \ldots, \Delta_{p}$ of $J$ such that (1) $m_{1}\left(\Delta_{i}\right)=2 r$, (2) the center $x_{i}$ of $\Delta_{i}$ belongs to $F_{k}$, (3) each point of $F_{k}$ belongs to at most two different $\Delta_{i}$, and (4) $p r<$ $m_{1}\left(F_{k}\right)+\varepsilon$. Choose $r \in(0, \delta)$ such that $|f(u)-f(v)|<t / 2$ whenever $|u-v| \leqq r \quad$ and $u, v \in Q$. Set $s_{i}=L\left(x_{i}, f, r\right), \quad V_{i}=B^{n}\left(f\left(x_{i}\right), s_{i}\right)$. Then $f F_{k} \subset \cup V_{i}$ and $d\left(V_{i}\right)=2 s_{i}<t$. Hence $\Lambda_{1}^{t}\left(f F_{k}\right) \leqq \sum d\left(V_{i}\right) \leqq$ $\sum 2 s_{i}$. By Hölder's inequality this implies

$$
\mathscr{A}_{1}^{t}\left(f F_{k}\right)^{n} \leqq 2^{n} p^{n-1} \sum s_{i}^{n}=\frac{2^{n} p^{n-1}}{\Omega_{n}} \sum m\left(V_{i}\right) .
$$

Since $x_{i} \in F_{k}$, we have $L^{*}\left(x_{i}, f, s_{i}\right) \leqq c l^{*}\left(x_{i}, f, s_{i}\right)=c r$, and therefore $U_{i}=U\left(x_{i}, f, s_{i}\right) \subset B^{n}\left(x_{i}, c r\right)$. Since $c r<1 / k$, this implies $V_{i}=f C_{i} \subset$ $f Z_{A}$ where $A=B^{n-1}(z, c r)$. Observing that every point in $Z_{A}$ belongs to at most $4 c$ different $U_{i}$, we get thus $\sum m\left(V_{i}\right) \leqq 4 c q m\left(f Z_{A}\right)=$ $4 c q \varphi(A, Q)$. From this and from $p r<m_{1}(F)+\varepsilon$ it follows

$$
\Lambda_{1}^{t}\left(f F_{k}\right)^{n} \leqq \frac{2^{n+2} c^{n} q\left(m_{1}(F)+\varepsilon\right)^{n-1} \Omega_{n-1} \varphi(A, Q)}{\Omega_{n} m_{n-1}(A)} .
$$

Letting first $r \rightarrow 0$, then $\varepsilon \rightarrow 0$, and then $t \rightarrow 0$, we obtain $\Lambda_{1}\left(f F_{k}\right)^{n} \leqq$ $q C_{\bar{\varphi}}^{\prime}(z, Q) m_{1}\left(F^{\prime}\right)^{n-1}$ where $C=2^{n+2} c^{n} \Omega_{n-1} / \Omega_{n}$. Since $f F$ is the limit of the expanding sequence of the compact sets $f F_{k}$, we have $\Lambda_{1}(f F)=$ $\lim \Lambda_{1}\left(f F_{k}\right)$ and hence

$$
\Lambda_{1}(f F)^{n} \leqq q C \bar{\varphi}^{\prime}(z, Q) m_{1}(F)^{n-1} .
$$


Let now $I_{j}, j=1, \ldots, m$, be disjoint closed subintervals of $J$. We have $\sum d\left(f I_{j}\right) \leqq \sum \Lambda_{1}\left(f I_{j}\right) \leqq q \Lambda_{1}\left(f \cup I_{j}\right)$. Applying (4.12) to $F=\cup I_{j}$ we obtain

$$
\left(\sum d\left(f I_{j}\right)\right)^{n} \leqq q^{n+1} C \bar{\varphi}^{\prime}(z, Q)\left(\sum m_{1}\left(I_{j}\right)\right)^{n-1}
$$

Thus (2.18) is true for $i=n$. By symmetry, it holds also for $1 \leqq i \leqq n-1$. Hence $f$ is $\mathrm{ACL}^{n}$.

We are now in a position to prove the metric definitions for quasiregular mappings.

4.13. Theorem. A non-constant mapping $f: G \rightarrow R^{n}$ is quasiregular if and only if it satisfies the following conditions:

(1) $f$ is sense-preserving, discrete, and open.

(2) $H(x, f)$ is locally bounded in $G$.

(3) There exists $a<\infty$ such that $H(x, f) \leqq a$ for almost every $x \in$ $G \backslash B_{f}$.

Proof. Suppose first that $f$ satisfies the conditions (1), (2), and (3). The mapping $f$ is $\mathrm{ACL}^{n}$ by 4.11. An open $\mathrm{ACL}^{n}$-mapping is differentiable a.e. [24, p. 9]. Let $D \in J(G)$. By (2) there exists $c<\infty$ such that $H(x, f)$ $\leqq c$ for $x \in D$. If $f$ is differentiable at $x \in D$, we have

$$
\left|f^{\prime}(x)\right|^{n} \leqq c^{n-1} J(x, f) .
$$

Since this holds a.e. in $D, f \mid D$ is quasiregular. If $x \in D \backslash B_{f}$ and if $U$ is a connected neighborhood of $x$ such that $U \subset D \backslash B_{f}$, it follows from (3) that $K_{o}(f \mid U), K_{I}(f \mid U) \leqq a^{n-1}$. Hence $f \mid D$ is $a^{n-1}$-quasiregular by 2.28. Since this is true for every $D \in J(G), f$ is $a^{n-1}$-quasiregular.

Let now $f$ be quasiregular. The condition (1) follows from 2.26, and (2) and (3) from 4.5 and 2.12.

4.14. Theorem. A non-constant mapping $f: G \rightarrow R^{n}$ is quasiregular if and only if it satisfies the following conditions:

(1) $f$ is sense-preserving, discrete, and open.

(2) $H^{*}(x, f)$ is locally bounded in $G$.

(3) There exists $a^{*}<\infty$ such that $H^{*}(x, f) \leqq a^{*}$ for almost every $x \in G \backslash B_{f}$.

Proof. Suppose that $f$ satisfies (1), (2), and (3). As in the proof of 4.13 we conclude that $f$ is $\mathrm{ACL}^{n}$ and differentiable a.e. Each point in $G \backslash B_{f}$ has a connected neighborhood $U$ such that $f \mid U$ is injective. Hence $H\left(f(z),(f \mid U)^{-1}\right)=H^{*}(z, f)$ for $z$ in such a $U$. This together with (2) and (3) imply that $(f \mid U)^{-1}$ and hence $f \mid U$ are $a^{* n-1}$-quasiconformal. If, 
in addition to this, we show that $\left|f^{\prime}(x)\right|=J(x, f)=0$ for almost every $x \in B_{f}$, we can conclude that $f$ is $a^{* n-1}$-quasiregular. Let $x_{0} \in B_{f}$ be a point where $f$ is differentiable. By 2.14, $J\left(x_{0}, f\right)=0$. Since $f$ is differentiable a.e., it is sufficient to show that $f^{\prime}\left(x_{0}\right)=0$. We have

$$
f\left(x_{0}+h\right)-f\left(x_{0}\right)=f^{\prime}\left(x_{0}\right) h+|h| \varepsilon(h)
$$

where $\varepsilon(h) \rightarrow 0$ as $h \rightarrow 0$. Suppose that $\left|f^{\prime}\left(x_{0}\right)\right|=\lambda>0$. Choose unit vectors $h_{1}, h_{2}$ such that $\left|f^{\prime}\left(x_{0}\right) h_{1}\right|=\lambda$ and $f^{\prime}\left(x_{0}\right) h_{2}=0$. Consider $r>0$ such that $U\left(x_{0}, f, r\right)$ is a normal neighborhood of $x_{0}$. Choose $\alpha_{1}, \alpha_{2}>0$ such that $x_{0}+\alpha_{i} h_{i} \in \partial U\left(x_{0}, f, r\right), i=1,2$. Then $\mid f\left(x_{0}+\alpha_{i} h_{i}\right)-$ $f\left(x_{0}\right) \mid=r$, and we obtain from (4.15)

$$
\left|\alpha_{1} f^{\prime}\left(x_{0}\right) h_{1}+\alpha_{1} \varepsilon\left(\alpha_{1} h_{1}\right)\right|=r=\alpha_{2}\left|\varepsilon\left(\alpha_{2} h_{2}\right)\right| .
$$

Thus

$$
\frac{L^{*}\left(x_{0}, f, r\right)}{l^{*}\left(x_{0}, f, r\right)} \geqq \frac{\alpha_{2}}{\alpha_{1}} \geqq \frac{\lambda-\left|\varepsilon\left(\alpha_{1} h_{1}\right)\right|}{\left|\varepsilon\left(\alpha_{2} h_{2}\right)\right|},
$$

which implies $H^{*}\left(x_{0}, f\right)=\infty$. By (2), this is a contradiction.

If $f$ is quasiregular, the conditions (1), (2), and (3) follow from 2.26, 4.6, and 2.12 .

\section{Condensers}

5.1. In this section we generalize the concept of a ring domain and its capacity. This generaljzation is called a condenser, and we state some properties of the capacity of a condenser.

5.2. Definition. A condenser is a pair $E=(A, C)$ where $A \subset R^{n}$ is open and $C$ is a non-empty compact set contained in $A . E$ is a ringlike condenser if $A \backslash C$ is a ring (see 2.1). $E$ is a bounded condenser if $A$ is bounded. A condenser $E=(A, C)$ is said to be in a domain $G$ if $A \subset G$.

The following lemma is immediate.

5.3. Lemma. If $f: G \rightarrow R^{n}$ is open and $E=(A, C)$ is a condenser in $G$, then $(f A, f C)$ is a condenser in $f G$.

In the above situation we denote $f E=(f A, f C)$.

5.4. The capacity of a condenser. Let $E=(A, C)$ be a condenser. We set

$$
\operatorname{cap} E=\operatorname{cap}(A, C)=\inf _{u \in W_{0}(E)} \int_{A}|\nabla u|^{n} d m
$$


and call it the capacity of the condenser $E$. The set $W_{0}(E)=W_{0}(A, C)$ is the family of all non-negative functions $u: A \rightarrow R^{1}$ such that (1) $u \in C_{0}(A)$, (2) $u(x) \geqq 1$ for $x \in C$, and (3) $u$ is ACL. In the above formula

$$
|\nabla u|=\left(\sum_{i=1}^{n}\left(\partial_{i} u\right)^{2}\right)^{1 / 2} .
$$

We mention some properties of the capacity of a condenser.

5.5. Lemma. If $E=(A, C)$ is a condenser, then

$$
\operatorname{cap} E=\inf _{u \in W_{0} \infty(E)} \int_{A}|\nabla u|^{n} d m
$$

where $W_{0}^{\infty}(E)=W_{0}^{\infty}(A, C)=W_{0}(E) \cap C_{0}^{\infty}(A)$.

Proof. Obviously

$$
\operatorname{cap} E \leqq \inf _{u \in W_{0}^{\infty}(E)} \int_{A}|\nabla u|^{n} d m .
$$

The converse inequality is proved by a standard approximating argument. The construction involves first multiplying $u \in W_{0}(E)$ by $1+\varepsilon, \varepsilon>0$, so that the resulting function is $\geqq(1+\varepsilon)$ on $C$, and then forming a smooth integral average, cf. e.g. [26, Section 27]. The details may be omitted.

5.6. Lemma. If $E=(A, C)$ is a ringlike condenser, then cap $E=$ $\operatorname{cap}(A \backslash C)$ in the sense of Gehring [8, p. 500].

This is a direct consequence of $n$-dimensional versions of $[8$, Lemma 1 , p. 501] and [8, Remark, p. 502].

5.7. Lemma. If $E=(A, C)$ is a condenser, then

$$
\operatorname{cap} E=\inf \operatorname{cap}(U, C) \text {, }
$$

where the infimum is taken over all open sets $U$ such that $\bar{U}$ is compact in $A$ and $U \supset C$.

Proof. Obviously $\operatorname{cap} E \leqq \operatorname{cap}(U, C)$ for all sets $U$ of the above type, hence

$$
\operatorname{cap} E \leqq \inf \operatorname{cap}(U, C) \text {. }
$$

Let $\varepsilon>0$. Then there exists a function $u \in W_{0}(E)$ such that 


$$
\operatorname{cap} E>\int_{A}|\nabla u|^{n} d m-\varepsilon
$$

Since spt $u$ is compact in $A$, there exists an open set $U$ such that spt $u$ $\subset U$ and $\bar{U}$ is compact in $A$. Then $u \in W_{0}(U, C)$ and we obtain

$$
\operatorname{cap}(U, C) \leqq \int_{A}|\nabla u|^{n} d m<\operatorname{cap}(A, C)+\varepsilon
$$

The lemma follows.

\subsection{Lemma. The inequality}

$$
\operatorname{cap} E \leqq \frac{m(A)}{d(C, \partial A)^{n}}
$$

holds for the capacity of a bounded condenser $E=(A, C)$.

Proof. Let $0<\varepsilon<d(C, \partial A)^{n}$. There exists an open set $U$ such that $C \subset U \subset \bar{U} \subset A$ and $d(C, \partial A)^{n} \leqq d(C, \partial U)^{n}+\varepsilon$. If we define $u(x)=$ $d(x, \mathbf{C} U) / d(C, \partial U)$, then $|u(x)-u(y)| \leqq|x-y| / d(C, \partial U)$ for all $x$, $y \in R^{n}$. Thus $u \in W_{0}(E)$ and $|\nabla u| \leqq 1 / d(C, \partial U)$ a.e., which implies

$$
\operatorname{cap} E \leqq \int_{A} d(C, \partial U)^{-n} d m=\frac{m(A)}{d(C, \partial U)^{n}} \leqq \frac{m(A)}{d(C, \partial A)^{n}-\varepsilon}
$$

Letting $\varepsilon \rightarrow 0$ gives the desired result.

5.9. Lemma. Suppose that $E=(A, C)$ is a condenser such that $C$ is connected. Then

$$
(\operatorname{cap} E)^{n-1} \geqq b_{n} \frac{d(C)^{n}}{m(A)}
$$

where $b_{n}$ is a positive constant which depends only on $n$.

Proof. By 5.7 we may suppose that $A$ is bounded. We may also assume that $d(C)=r>0$ and that $C$ contains the origin and the point $r e_{n}$. Let $u \in W_{0}^{\infty}(E)$. For $0<t<r$ we let $T(t)$ denote the hyperplane $x_{n}=t$. Using the method of $[23$, p. 9$]$, we estimate the integral

$$
\int_{T(t)}|\nabla u|^{n} d m_{n-1} \text {. }
$$


Fix $z \in C \cap T(t)$. For $y \in S^{n-2}$ let $R(y)$ be the supremum of all $t_{0}>0$ such that $z+t y \in A$ for $0 \leqq t<t_{0}$. Then

$$
\int_{0}^{R(y)}|\nabla u(z+t y)| d t \geqq u(z)-u(z+R(y) y) \geqq 1
$$

for all $y \in S^{n-2}$. By Hölder's inequality this implies

$$
1 \leqq(n-1)^{n-1} R(y) \int_{0}^{R(y)}|\nabla u(z+t y)|^{n} t^{n-2} d t .
$$

Integrating over $y \in S^{n-2}$ yields

$$
\begin{gathered}
(n-1)^{1-n} \int_{S^{n-2}} R^{-1} d m_{n-2} \\
\leqq \int_{S^{n-2}} d m_{n-2}(y) \int_{0}^{R(y)}|\nabla u(z+t y)|^{n} t^{n-2} d t \\
\leqq \int_{T(t)}|\nabla u|^{n} d m_{n-1} .
\end{gathered}
$$

On the other hand, we obtain by Hölder's inequality

$$
\begin{aligned}
\omega_{n-2}^{n} & =\left(\int_{S^{n-2}} d m_{n-2}\right)^{n} \leqq \int_{S^{n-2}} R^{n-1} d m_{n-2}\left(\int_{S^{n-2}} R^{-1} d m_{n-2}\right)^{n-1} \\
& \leqq(n-1) m_{n-1}(A \cap T(t))\left(\int_{S^{n-2}} R^{-1} d m_{n-2}\right)^{n-1} .
\end{aligned}
$$

Setting $f(t)=m_{n-1}(A \cap T(t))$, we obtain from (5.10) and (5.11)

$$
\int_{T(t)}|\nabla u|^{n} d m_{n-1} \geqq(n-1)^{1-n-1 /(n-1)} \omega_{n-2}^{n /(n-1)} f(t)^{1 /(1-n)} .
$$

Integrating over $0<t<r$ we obtain

$$
\int_{A}|\nabla u|^{n} d m \geqq(n-1)^{1-n-1 /(n-1)} \omega_{n-2}^{n /(n-1)} \int_{0}^{r} f(t)^{1 /(1-n)} d t .
$$

Hölder's inequality gives

$$
r^{n}=\left(\int_{0}^{r} d t\right)^{n} \leqq\left(\int_{0}^{r} f(t) d t\right)\left(\int_{0}^{r} f(t)^{1 /(1-n)} d t\right)^{n-1} \leqq m(A)\left(\int_{0}^{r} f(t)^{1 /(1-n)} d t\right)^{n-1}
$$


By (5.12), this implies

$$
\left(\int_{A}|\nabla u|^{n} d m\right)^{n-1} \geqq(n-1)^{-2+2 n-n^{2}} \omega_{n-2}^{n} \frac{r^{n}}{m(A)} .
$$

Since this holds for every $u \in W_{0}^{\infty}(E)$, the lemma follows.

\section{The condenser definition for $K_{o}(f)$}

6.1. Suppose that $f: G \rightarrow R^{n}$ is a mapping. A condenser $E=(A, C)$ is said to be a normal condenser of $f$ if $A$ is a normal domain of $f$. If $E$ is a normal condenser, we set $N(f, E)=N(f, A)=\mu(f, A)$ (cf. 2.4).

This section is devoted to the proof of the following result:

6.2. Theorem. Suppose that $f: G \rightarrow R^{n}$ is sense-preserving, discrete, and open, and that $1 \leqq K<\infty$. Then the following conditions are equivalent:

(1) $K_{o}(f) \leqq K$.

(2) cap $E \leqq K N(f, E)$ cap $f E$ for all normal contensers $E$ in $G$.

(3) $\operatorname{cap} E \leqq K N(f, E) \operatorname{cap} f E$ for all ringlike normal condensers $E$ in $G$.

Since (2) implies (3) trivially, it suffices to prove that $(3) \Rightarrow(1) \Rightarrow(2)$.

6.3. Proof for $(3) \Rightarrow(1)$. We show first, using the metric definition 4.13, that $f$ is quasiregular. Let $x \in G$, and choose $\sigma_{x}>0$ as in 2.9 . Choose $0<r_{1}<r_{2}<\sigma_{x}$, and set $U_{i}=U\left(x, f, r_{i}\right), i=1,2$. Then $E=\left(U_{2}, \bar{U}_{1}\right)$ is a ringlike normal condenser. Let $\Gamma$ be the family of all paths joining $\partial U_{1}$ and $\partial U_{2}$ in $U_{2} \backslash \bar{U}_{1}$, and let $\Gamma_{1}$ be the family of all paths joining the boundary components of the spherical ring $A=B^{n}\left(f(x), r_{2}\right) \backslash$ $\left.\bar{B}^{n}\left(f(x), r_{1}\right)\right) \quad$ in $A$. By $2.9, \quad \partial U_{i}=\bar{U}_{2} \cap f^{-1} S^{n-1}\left(f(x), r_{i}\right), \quad i=1,2$. From the path lifting lemma 2.7 it follows that $\Gamma_{1}=f \Gamma$.

By 5.6 and by a generalized version of [10, Theorem 1], cap $E=M(T)$ and $\operatorname{cap} f E=M\left(\Gamma_{1}\right)$. Hence (3) implies $M(\Gamma) \leqq K i(x, f) M(f \Gamma)$. From 4.4 it follows that $H(x, f) \leqq C<\infty$ where $C$ depends only on $n, K$, and $i(x, f)$. Since $i(x, f)=1$ for $x \in G \backslash B_{f}$, it follows from 4.13 that $f$ is quasiregular.

If $x \in G \backslash B_{f}$, then there is a neighborhood $V=B^{n}(x, r)$ such that $f \mid V$ is injective. By (3) and by 5.6, cap $R \leqq K$ cap $f R$ for all rings $R \subset V$. Hence $K_{o}(f \mid V) \leqq K$ by the corresponding result for quasiconformal mappings [26, 36.1]. By 2.28, $K_{o}(f) \leqq K$.

6.4. Proof for $(1) \Rightarrow(2)$. Suppose that $E=(A, C)$ is a normal condenser in $G$. Let $\varepsilon>0$. By 5.5, there is $v \in W_{0}^{\infty}(f E)$ such that 


$$
\int_{f A}|\nabla v|^{n} d m<\operatorname{cap} f E+\varepsilon .
$$

Define $u: A \rightarrow R^{1}$ by $u(x)=v(f(x))$. Then $u(x) \geqq 1$ for $x \in C$. Since $f$ is ACL and a.e. differentiable, $u$ has also these properties. Since $A$ is a normal domain of $f$, it follows from 2.6 that spt $u \subset A \cap f^{-1}$ (spt $\left.v\right)$ is compact. Hence $u \in W_{0}(E)$, which implies

$$
\operatorname{cap} E \leqq \int_{A}|\nabla u|^{n} d m \text {. }
$$

Here $|\nabla u(x)| \leqq|\nabla v(f(x))|\left|f^{\prime}(x)\right|$ a.e. Using [14, Theorem 3, p. 364] we obtain

$$
\begin{gathered}
\operatorname{cap} E \leqq K_{o}(f) \int_{A}|\nabla v(f(x))|^{n} J(x, f) d m(x) \\
=K_{o}(f) \int_{R^{n}}|\nabla v(y)|^{n} N(y, f, A) d m(y) \\
\leqq N(f, E) K_{o}(f) \int_{f_{A}}|\nabla v|^{n} d m \leqq N(f, E) K_{o}(f)(\operatorname{cap} f E+\varepsilon) .
\end{gathered}
$$

Since $\varepsilon$ is arbitrary, (2) follows.

\section{The condenser definition for $K_{I}(f)$}

This section is devoted to the proof of the following result:

7.1. Theorem. Suppose that $f: G \rightarrow R^{n}$ is sense-preserving, discrete, and open, and that $1 \leqq K<\infty$. Then the following conditions are equivalent:

(1) $K_{I}(f) \leqq K$.

(2) $\operatorname{cap} f E \leqq K$ cap $E$ for all condensers $E$ in $G$.

(3) $\operatorname{cap} f E \leqq K$ cap $E$ for all ringlike condensers $E$ in $G$.

7.2. Remarks. This result differs from 6.2 in two respects. First, in 7.1 the factor $N(f, E)$ does not appear. Second, the inequality (2) holds for all condensers while the corresponding inequality cap $E \leqq N(f, E) K_{O}(f)$ cap $f E$ of 6.2 is given only for normal condensers. In particular, (2) holds for condensers $E=(A, C)$ such that $\bar{A}$ is not compact in $G$. This makes 7.1 to a useful tool when the boundary behavior of quasiregular mappings is studied. For example, suppose that $f: R^{n} \rightarrow R^{n}$ is a bounded non-constant quasiregu- 
lar mapping. If $E$ is the condenser $\left(R^{n}, \bar{B}^{n}\right)$, we have cap $E=0$, while 5.9 implies cap $f E>0$. This contradicts (2), and we have proved Liouville's theorem in $n$ dimensions (cf. Rešetnjak [19, p. 661]). We intend to return to related questions in a later paper. The proof of 7.1 is considerably more difficult than that of 6.2 . Since (2) implies (3) trivially, it suffices to prove that $(3) \Rightarrow(1) \Rightarrow(2)$.

7.3. Proof for $(3) \Rightarrow(1)$. We show first with the help of Lemma 2.17 that $f$ is $\mathrm{ACL}^{n}$. Let $D \in J(G)$, and let $Q$ be an open $n$-interval in $D$. Write $Q=Q_{0} \times J$, where $Q_{0}$ is an $(n-1)$-interval in $R^{n-1}$ and $J$ is an open segment of the $x_{n}$-axis. Using the notation of 2.16 , we have $\varphi_{n}(E, Q)$ $=m(f(E \times J))$ for Borel sets $E \subset Q_{0}$. Fix $z \in Q_{0}$ such that $\bar{\varphi}_{n}^{\prime}(z, Q)$ $<\infty$, and let $\Delta_{1}, \ldots, \Delta_{k}$ be disjoint closed subintervals of the segment $J_{z}=\{z\} \times J$. Set $A_{i}=\Delta_{i}+r B^{n}$ where $r$ is a positive number such that (i) the domains $A_{i}$ are disjoint, (ii) $A_{i} \subset Q$, and (iii) $\Omega_{n} r \leqq \omega_{n-1} m_{1}\left(A_{i}\right)$ for $1 \leqq i \leqq k$. Then $\left(A_{i}, \Delta_{i}\right)$ is a ringlike condenser, and we obtain from 5.8 and (iii) the estimate

$$
\operatorname{cap}\left(A_{i}, \Delta_{i}\right) \leqq m\left(A_{i}\right) / r^{n} \leqq 2 \omega_{n-1} m_{1}\left(\Delta_{i}\right) / r .
$$

On the other hand, 5.9 implies

$$
\left(\operatorname{cap}\left(f A_{i}, f \Delta_{i}\right)\right)^{n-1} \geqq b_{n} \frac{d\left(f \Delta_{i}\right)^{n}}{m\left(f A_{i}\right)} .
$$

Together with (3) these yield

$$
\left.d\left(f \Delta_{i}\right) \leqq C r^{(1-n) n} m\left(f A_{i}\right)^{1 n} m_{1}(\lrcorner_{i}\right)^{(n-1) n},
$$

where $C$ depends only on $n$ and $K$.

Summing over $1 \leqq i \leqq k$ and using Hölder's inequality we obtain

$$
\left(\sum_{i=1}^{k} d\left(f \Delta_{i}\right)\right)^{n} \leqq C_{1} m_{n-1}(B)^{-1}\left(\sum_{i=1}^{k} m\left(f A_{i}\right)\right)\left(\sum_{i=1}^{k} m_{1}\left(A_{i}\right)\right)^{n-1}
$$

where $B=B^{n-1}(z, r)$ and $C_{1}$ depends only on $n$ and $K$. Setting $q=N(f, D)$ we have $\sum m\left(f A_{i}\right) \leqq q m\left(\cup f A_{i}\right) \leqq q \varphi_{n}(B, Q)$. Letting $r \rightarrow 0$ we thus obtain

$$
\left(\sum_{i=1}^{k} d\left(f \Delta_{i}\right)\right)^{n} \leqq q C_{1} \bar{\varphi}_{n}^{\prime}(z, Q)\left(\sum_{i=1}^{k} m_{1}\left(\Delta_{i}\right)\right)^{n-1} .
$$

By symmetry, Lemma 2.17 implies that $f$ is $\mathrm{ACL}^{n}$.

As an open $\mathrm{ACL}^{n}$-mapping, $f$ is differentiable a.e. by $[24$, p. 9]. We prove that $f$ is quasiregular by showing that if $f$ is differentiable at $x_{0}$ : then

$$
\left|f^{\prime}\left(x_{0}\right)\right|^{n} \leqq K_{1} J\left(x_{0}, f\right)
$$


where $K_{1}$ depends only on $n$ and $K$. For brevity, we set $L=\left|f^{\prime}\left(x_{0}\right)\right|$ and $J=J\left(x_{0}, f\right)$. We may assume that $L>0$. Let $0<\varepsilon<L$, and let $e$ be a unit vector such that $\left|f^{\prime}\left(x_{0}\right) e\right|=L$. For $0<r<d\left(x_{0}, \partial G\right) / 2$ let $C_{r}$ be the closed line segment with end points $x_{0}$ and $x_{0}+r e$. Setting $A_{r}=C_{r}+r B^{n}$ we obtain a ringlike condenser $E_{r}=\left(A_{r}, C_{r}\right)$ in $G$. Since the condensers $E_{r}$ are similar for all $r$, cap $E_{r}=c$ is a positive constant, independent of $r$. We choose $r$ so small that $\left|f\left(x_{0}+r e\right)-f\left(x_{0}\right)\right|>(L-\varepsilon) r$ and $m\left(f A_{r}\right) \leqq(J+\varepsilon) m\left(A_{r}\right)$. Since $m\left(A_{r}\right) \leqq 3 \omega_{n-1} r^{n}$, 5.9 implies

$$
\left(\operatorname{cap} f E_{r}\right)^{n-1} \geqq b_{n} \frac{d\left(f C_{r}\right)^{n}}{m\left(f A_{r}\right)} \geqq C \frac{(L-\varepsilon)^{n}}{J+\varepsilon}
$$

where $C$ depends only on $n$. Since $\operatorname{cap} f E_{r} \leqq K \operatorname{cap} E_{r}=K c$, we obtain

$$
(L-\varepsilon)^{n} \leqq C^{-1} K^{n-1} c^{n-1}(J+\varepsilon) .
$$

Letting $\varepsilon \rightarrow 0$ yields (7.4). Hence $f$ is quasiregular.

Let $x \in G \backslash B_{f}$, and choose a connected neighborhood $D$ of $x$ such that $f \mid D$ is injective. Applying Theorem 6.2 to the mapping $(f \mid D)^{-1}$ we obtain

$$
K_{I}(f \mid D)=K_{o}\left((f \quad D)^{-1}\right) \leqq K .
$$

By $2.28, K_{I}(f) \leqq K$.

7.5. We now turn to the proof of $(1) \Rightarrow(2)$ in Theorem 7.1. Assume that $K_{I}(f) \leqq K$, and let $E=(A, C)$ be a condenser in $G$. Let $u$ be an arbitrary function in $W_{0}^{\infty}(E)$. We define $v: f A \rightarrow R^{1}$ by

$$
v(y)=\sup _{x \in f^{-1}(y)} u(x) \text {. }
$$

Since $f$ is discrete and since spt $u$ is compact, $f^{-1}(y) \cap$ spt $u$ is finite. Hence for each $y \in f A$ there is $x \in f^{-1}(y)$ such that $v(y)=u(x)$. We are going to show that $v \in W_{0}(f E)$. For this purpose we prove some lemmas.

7.6. Lemma. The function $v$ has the following properties:

(1) $v(y) \geqq 1$ for $y \in f C$.

(2) $\operatorname{spt} v=f(\operatorname{spt} u)$.

(3) spt $v$ is compact in $f A$.

(4) $v$ is continuous.

Proof. The property (1) is trivial. For (2), set $U=\{x \in A \mid u(x) \neq 0\}$ and $V=\{y \in f A \mid v(y) \neq 0\}$. Then $f U=V$. Since $f$ is continuous and spt $u=\bar{U}$ is compact, $f(\operatorname{spt} u)=\bar{V}=\operatorname{spt} v$. Since (3) is a consequence of (2), it remains to verify (4). 
Let $y \in f A$. If $y \notin \operatorname{spt} v$, then $v=0$ in a neighborhood of $y$. Next assume $y \in \operatorname{spt} v=f(\operatorname{spt} u)$. Let $\varepsilon>0$. Choose a neighborhood $U$ of spt $u$ such that $\bar{U}$ is a compact subset of $A$ and such that $\partial U \cap$ $f^{-1}(y)=\varnothing$. Let $U \cap f^{-1}(y)=\left\{x_{1}, \ldots, x_{j}\right\}$, where $u\left(x_{1}\right)=v(y)$. Вy the continuity of $u$ we can find neighborhoods $U\left(x_{i}\right)$ of $x_{i}, 1 \leqq i \leqq j$, such that $\left|u(x)-u\left(x_{i}\right)\right|<\varepsilon$ for $x \in U\left(x_{i}\right)$. Then $F=\bar{U} \backslash \bigcup_{i=1} U\left(x_{i}\right)$
is compact, $y \notin f F$, and

$$
V=\left(\bigcap_{i=1}^{j} f U\left(x_{i}\right)\right) \backslash f F
$$

is a neighborhood of $y$. We show that $|v(z)-v(y)|<\varepsilon$ if $z \in V$.

Since $z \in f U\left(x_{1}\right), z=f\left(x_{0}\right)$ for some $x_{0} \in U\left(x_{1}\right)$. Hence

$$
v(z) \geqq u\left(x_{0}\right) \geqq u\left(x_{1}\right)-\varepsilon=v(y)-\varepsilon .
$$

On the other hand, $v(z)=u(x)$ for some $x$ in $U \cap f^{-1}(z) \subset U \backslash F$. Hence $x \in U\left(x_{i}\right)$ for some $i$, which implies

$$
v(z)=u(x)<u\left(x_{i}\right)+\varepsilon \leqq v(y)+\varepsilon .
$$

The lemma is proved.

7.7. To show that $v \in W_{0}(f E)$, we still must prove that $v$ is ACL. Since this property is local, it suffices to show that $v$ is ACL in a neighborhood of each point of spt $v$. Fix $y_{0} \in \operatorname{spt} v$, and let $f^{-1}\left(y_{0}\right) \cap$ spt $u=$ $\left\{x_{1}, \ldots, x_{q}\right\}$. Choose $r_{0}$ such that $0<r_{0}<d\left(y_{0}, \partial f A\right)$ and such that the domains $U\left(x_{i}, f, r_{0}\right)$ are disjoint normal neighborhoods of $x_{i}$ for $1 \leqq i \leqq q$ (see 2.9). Next choose a positive number $r_{1} \leqq r_{0}$ such that $B^{n}\left(y_{0}, r_{1}\right) \cap f\left(\operatorname{spt} u \backslash \bigcup_{i=1}^{q} U\left(x_{i}, f, r_{0}\right)\right)=\varnothing$. Then the components of $f^{-1} B^{n}\left(y_{0}, r_{1}\right)$ which meet spt $u$ are the sets $U\left(x_{i}, f, r_{1}\right), 1 \leqq i \leqq q$. Set $U_{i}=U\left(x_{i}, f, r_{1}\right)$ and $U=\bigcup_{i=1}^{q} U_{i}$. Choose an open $n$-interval $Q$ such that $\bar{Q} \subset B^{n}\left(y_{0}, r_{1}\right)$. Write $Q=Q_{0} \times J$, where $Q_{0}$ is an $(n-1)$ interval in $R^{n-1}$, and $J=(a, b)$ is an open segment of the $x_{n}$-axis. For each Borel set $A \subset Q_{0}$ put $\varphi(A)=m\left(U \cap f^{-1}(A \times J)\right)$. Then $\varphi$ is a 1-quasiadditive (in fact, completely additive) set function in $Q_{0}$. By 2.3, $\bar{\varphi}^{\prime}(z)<\infty$ for almost every $z \in Q_{0}$. Fix such $z$, and set $J_{z}=\{z\}$ $\times J$. To show that $v$ is ACL, it is sufficient to prove the following result:

7.8. Lemma. The function $v$ is absolutely continuous on $\bar{J}_{z}$.

7.9. For the proof of 7.8 we need another lemma, which states, roughly speaking, that the one-to-many correspondence $f^{-1}$ is absolutely conti- 
nuous on $\bar{J}_{z}$. We let $\Phi$ denote the set of all continuous mappings $g: \bar{J}_{z} \rightarrow U$ such that $f \circ g$ is the identity mapping of $\bar{J}_{z}$. Observe that for any $g \in \Phi, g \bar{J}_{z}$ is contained in some $U_{i}$.

7.10. Lemma. For every $\varepsilon>0$ there is $\delta>0$ such that

$$
\sum_{i=1}^{p}\left|g_{i}\left(\bar{y}_{i}\right)-g_{i}\left(y_{i}\right)\right|<\varepsilon
$$

whenever $\left[y_{1}, \bar{y}_{1}\right], \ldots,\left[y_{p}, \bar{y}_{p}\right]$ are disjoint closed intervals of $J_{z}$, $\sum_{i=1}^{p}\left|\bar{y}_{i}-y_{i}\right|<\delta$, and $g_{i} \in \Phi, 1 \leqq i \leqq p$.

Proof. The proof is closely related to the proof of 4.11. By 4.6, there is a constant $c$ such that $H^{*}(x, f)<c$ for $x \in U$. Suppose that $\left[y_{i}, \bar{y}_{i}\right]$

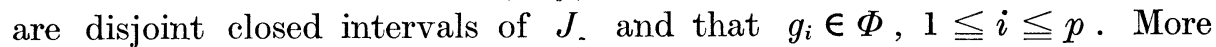
precisely, $y_{i}=\left(z, t_{i}\right), \bar{y}_{i}=\left(z, \bar{t}_{i}\right)$, and $a<t_{1}<\bar{t}_{1}<\ldots<t_{p}<\bar{t}_{p}<b$. Set $F^{i}=\left[y_{i}, \bar{y}_{i}\right]$ and $F=F^{1} \cup \ldots \cup F^{p}$. Choose an integer $k_{0}$ such that $0<1 / k_{0}<d(F, \partial Q)$. For $k \geqq k_{0}$ and for $1 \leqq i \leqq p$ let $F_{k}^{i}$ denote the set of all points $y \in F^{i}$ such that $0<r<1 / k$ implies $L^{*}\left(g_{i}(y), f, r\right) \leqq c l^{*}\left(g_{i}(y), f, r\right)$. Then $F_{k}^{i} \subset F_{k+1}^{i}$ and $F^{i}=\bigcup_{k=k_{0}}^{\infty} F_{k}^{i}$. By 4.8, the function $y \mapsto L^{*}\left(g_{i}(y), f, r\right)$ is lower semicontinuous, and the function $y \mapsto l^{*}\left(g_{i}(y), f, r\right)$ is continuous. Hence each $F_{k}^{i}$ is compact.

Let $\eta$ and $t$ be arbitrary positive numbers, and fix $k \geqq k_{0}$. Using the same lemma as in the proof of 4.11 ([7, p. 6] or $[26,31.1])$, we can find positive numbers $\delta_{1}, \ldots, \delta_{p}$ such that for all $r \in\left(0, \delta_{i}\right)$ there exists a covering of $F_{k}^{i}$ by open intervals $\Delta_{1}^{i}, \ldots, \Delta_{l(i)}^{i}$ such that $(1) m_{1}\left(\Delta_{m}^{i}\right)=2 r$, (2) the center $y_{m}^{i}$ of $\Delta_{m}^{i}$ belongs to $F_{k}^{i}$, (3) every point of $F_{k}^{i}$ belongs to at most two different $\Delta_{m}^{i}$, and (4) $l(i) r<m_{1}\left(F_{k}^{i}\right)+\eta / p$. Set $\alpha=\min _{1 \leqq i \leqq p-1}$ $\left|y_{i+1}-\bar{y}_{i}\right|$. Choose $r>0$ such that $r \leqq \min \left(\delta_{1}, \ldots, \delta_{p}, 1 / k, \alpha / 2\right)$ and such that $\left|g_{i}(y)-g_{i}\left(y^{\prime}\right)\right|<t / 2 c$ whenever $\left|y-y^{\prime}\right| \leqq 2 r, y, y^{\prime} \in \bar{J}_{z}$, and $1 \leqq i \leqq p$. Then $\left\{\Delta_{m}^{i} \mid 1 \leqq i \leqq p, 1 \leqq m \leqq l(i)\right\}$ is a covering of $\bigcup_{i=1}^{P} F_{k}^{i}$ such that every point is covered at most twice.

Set $x_{m}^{i}=g_{i}\left(y_{m}^{i}\right) \quad$ and $\quad U_{m}^{i}=U\left(x_{m}^{i}, f, r\right), 1 \leqq i \leqq p, 1 \leqq m \leqq l(i)$. Since $y_{m}^{i} \in F_{k}^{i}$, we have $L_{i m}^{*} \leqq c l_{i m}^{*}$ where $L_{i m}^{*}=L^{*}\left(x_{m}^{i}, f, r\right), l_{i m}^{*}=$ $l^{*}\left(x_{m}^{i}, f, r\right)$. On the other hand, $l_{\iota m}^{*} \leqq d\left(g_{i} \Delta_{m}^{i}\right)<t / 2 c$, which implies $d\left(U_{m}^{i}\right) \leqq 2 L_{i m}^{*}<t$. Since $g_{i} \Delta_{m}^{i} \subset U_{m}^{i}$, this yields

$$
\Lambda_{1}^{t}\left(\bigcup_{i=1}^{p} g_{i} F_{k}^{i}\right)^{n} \leqq \sum_{i=1}^{p} \sum_{m=1}^{l(i)} d\left(U_{m}^{i}\right) \leqq 2 c \sum_{i=1}^{p} \sum_{m=1}^{l(i)} l_{i m}^{*} .
$$


By Hölder's inequality we obtain

$$
\Lambda_{1}^{t}\left(\bigcup_{i=1}^{p} g_{i} F_{k}^{i}\right)^{n} \leqq 2^{n} c^{n}\left(\sum_{i=1}^{p} \sum_{m=1}^{l(i)} l_{i m}^{* n}\right)\left(\sum_{i=1}^{p} l(i)\right)^{n-1} .
$$

Since $l(i) r \leqq m_{1}\left(F_{k}^{i}\right)+\eta / p \leqq m_{1}\left(F^{i}\right)+\eta / p$ and since $\Omega_{n} l_{i m}^{* n} \leqq m\left(U_{m}^{i}\right)$, this implies

$$
\Lambda_{1}^{t}\left(\bigcup_{i=1}^{p} g_{i} F_{k}^{i}\right)^{n} \leqq \frac{2^{n} c^{n}\left(m_{1}(F)+\eta\right)^{n-1} \sum \sum m\left(U_{m}^{i}\right)}{\Omega_{n} r^{n-1}} .
$$

Set $B=B^{n-1}(z, r)$. Then each $U_{m}^{i}$ is contained in $U \cap f^{-1}(B \times J)$. Since every point belongs to not more than two different $U_{m}^{i}$, we have $\sum \sum m\left(U_{m}^{i}\right) \leqq 2 m\left(U \cap f^{-1}(B \times J)\right)=2 \varphi(B)$. Thus

$$
A_{1}^{t}\left(\bigcup_{i=1}^{p} g_{i} F_{k}^{i}\right)^{n} \leqq C\left(m_{1}(F)+\eta\right)^{n-1} \varphi(B) / m_{n-1}(B)
$$

where $C=2^{n+1} c^{n} \Omega_{n-1} / \Omega_{n}$. Letting first $r \rightarrow 0$, then $\eta \rightarrow 0$, then $t \rightarrow 0$, and then $k \rightarrow \infty$ yields

$$
\left(\sum_{i=1}^{p} A_{1}\left(g_{i} F^{i}\right)\right)^{n}=A_{1}\left(\bigcup_{i=1}^{p} g_{i} F^{i}\right)^{n} \leqq C \bar{\varphi}^{\prime}(z) m_{1}(F) .
$$

Since $\left|g_{i}\left(\bar{y}_{i}\right)-g_{i}\left(y_{i}\right)\right| \leqq A_{1}\left(g_{i} F^{i}\right)$, this proves 7.10 .

7.11. Proof for Lemma 7.8. We show first that for every pair $y, \bar{y} \in J_{z}$ there are $g, \bar{g} \in \Phi$ such that

$$
|v(\bar{y})-v(y)| \leqq|u(g(\bar{y}))-u(g(y))|+|u(\bar{g}(\bar{y}))-u(\bar{g}(y))| \cdot
$$

By 2.7, there are $g, \bar{g} \in \Phi$ such that $v(y)=u(g(y))$ and $v(\bar{y})=u(\bar{g}(\bar{y}))$. If $v(y) \leqq v(\bar{y})$, then

$$
|v(\bar{y})-v(y)|=u(\bar{g}(\bar{y}))-u(g(y)) \leqq u(\bar{g}(\bar{y}))-u(\bar{g}(y)),
$$

and if $v(\bar{y}) \leqq v(y)$, then

$$
|v(\bar{y})-v(y)|=u(g(y))-u(\bar{g}(\bar{y})) \leqq u(g(y))-u(g(\bar{y})) .
$$

These inequalities prove (7.12).

Let $\varepsilon>0$, and let $\delta>0$ be the number given by 7.10. Since $u \in$ $C_{0}^{\infty}(A), u$ satisfies a Lipschitz condition.

$$
\left|u(x)-u\left(x^{\prime}\right)\right| \leqq M\left|x-x^{\prime}\right|
$$

for all $x, x^{\prime} \in A$. Let $\left[y_{1}, \bar{y}_{1}\right], \ldots,\left[y_{p}, \bar{y}_{p}\right]$ be disjoint closed intervals of $J_{z}$ such that $\sum\left|\bar{y}_{i}-y_{i}\right|<\delta$. By (7.12) there are $g_{i}, \bar{g}_{i} \in \Phi$ such that

$$
\left|v\left(\bar{y}_{i}\right)-v\left(y_{i}\right)\right| \leqq\left|u\left(g_{i}\left(\bar{y}_{i}\right)\right)-u\left(g_{i}\left(y_{i}\right)\right)\right|+\left|u\left(\bar{g}_{i}\left(\bar{y}_{i}\right)\right)-u\left(\bar{g}_{i}\left(y_{i}\right)\right)\right|
$$


for $1 \leqq i \leqq p$. By 7.10 and (7.13) we obtain

$$
\sum_{i=1}^{p}\left|v\left(\bar{y}_{i}\right)-v\left(y_{i}\right)\right| \leqq 2 M \varepsilon .
$$

Hence $v$ is absolutely continuous on $\bar{J}_{z}$, and 7.8 is proved. This implies:

7.14. Lemma. $v \in W_{0}(f E)$.

7.15. Lemma. Suppose that $y \in \operatorname{spt} v \backslash f\left(\operatorname{spt} u \cap B_{f}\right)$. Then there is a neighborhood $V_{0}$ of $y$ such that for every connected neighborhood $V \subset V_{0}$ of $y$, the following conditions are satisfied:

(1) $V \cap f\left(B_{f} \cap \operatorname{spt} u\right)=\varnothing$.

(2) The components of $f^{-1} V$ which meet spt $u$ form a finite collection $D_{1}, \ldots, D_{k}$.

(3) $f$ defines homeomorphisms $f_{i}: D_{i} \rightarrow V$.

(4) $v$ is differentiable a.e. in $V$.

(5) $|\nabla v(z)| \leqq \max _{1 \leqq i \leqq k}\left|\nabla u\left(g_{i}(z)\right)\right|\left\{g_{i}^{\prime}(z) \mid\right.$ for almost every $z \in V$ where $g_{i}=f_{i}^{-1}$.

Proof. Choose disjoint neighborhoods $U_{1}, \ldots, U_{k}$ of the points of spt $u \cap f^{-1}(y)$ such that $U_{i} \in J(G)$ and such that $f \mid \bar{U}_{i}$ is injective, $1 \leqq i \leqq k$. We claim that

$$
V_{0}=\left(\bigcap_{i=1}^{k} f U_{i}\right) \backslash f\left(\operatorname{spt} u \backslash \bigcup_{i=1}^{k} U_{i}\right)
$$

is the required neighborhood of $y$.

Let $V \subset V_{0}$ be a connected neighborhood of $y$. Then (1) holds since $U_{i} \cap B_{f}=\varnothing$ for $1 \leqq i \leqq k$. If $D$ is a component of $f^{-1} V$ such that $D \cap$ spt $u \neq \varnothing$, then $D$ meets some $U_{i}$. Since $f \mid \bar{U}_{i}$ is injective, we have $V_{0} \cap f \partial U_{i}=\varnothing$, and hence $D \cap \partial U_{i}=\varnothing$. This implies $D \subset U_{i}$, which proves (2) and (3).

Since the mappings $g_{i}$ are quasiconformal, they are differentiable a.e. in $V$. Let $z \in V$ be a point at which every $g_{i}, 1 \leqq i \leqq k$, is differentiable. Let $I$ be the set of all indexes $i$ such that $v(z)=u\left(g_{i}(z)\right)$. If $h \in R^{n}$ is small enough, then by the continuity of $v, v(z+h)=\max _{j \in I} u\left(g_{j}(z+h)\right)$. Thus

$$
|v(z+h)-v(z)|=\left(u\left(g_{j}(z+h)\right)-u\left(g_{j}(z)\right) \mid\right.
$$

for some $j \in I$. By (7.13), this yields

$$
|v(z+h)-v(z)| \leqq M \max _{1 \leqq i \leqq k}\left|g_{i}(z+h)-g_{i}(z)\right| .
$$


Since the mappings $g_{i}$ are differentiable at $z$, this implies

$$
\limsup _{h \rightarrow 0} \frac{|v(z+h)-v(z)|}{|h|}<\infty,
$$

and (4) follows from the theorem of Rademacher and Stepanov [26, 29.1]. Finally, (5) follows easily from (7.16).

7.17. We shall now complete the proof of Theorem 7.1. Let $u \in W_{0}^{\infty}(E)$ and $v$ be as in 7.5. By Lemma 7.14 we have

$$
\operatorname{cap} f E \leqq \int_{f A}|\nabla v|^{n} d m .
$$

There exists a countable net of open disjoint cubes $Q_{1}, Q_{2}, \ldots$ such that $f A \backslash f\left(\operatorname{spt} u \cap B_{f}\right)=\bigcup_{j=1}^{\infty} \bar{Q}_{j}$ and such that if $Q_{j}$ meets spt $v$, then the conditions $(1)-(5)$ of 7.15 are satisfied for $V=Q_{j}$. Since $m\left(f B_{f}\right)=0$ by $2.27, m\left(f A \backslash \bigcup_{j=1}^{\infty} Q_{j}\right)=0$. Hence

$$
\operatorname{cap} f E \leqq \sum_{j=1}^{\infty} \int_{Q_{j}}|\nabla v|^{n} d m .
$$

Consider a fixed cube $Q_{j}$. If $Q_{j}$ does not meet $\operatorname{spt} v$, then

$$
\int_{Q_{j}}|\nabla v|^{n} d m=0 \text {. }
$$

If $Q_{j}$ meets spt $v$, consider the inverse mappings $g_{i}: Q_{j} \rightarrow D_{i}, \quad 1 \leqq i$ $\leqq k$, given by 7.15 . For almost every $z \in Q_{j}$ we have

$$
\begin{aligned}
|\nabla v(z)|^{n} & \leqq \max _{1 \leqq i \leqq k}\left|\nabla u\left(g_{i}(z)\right)\right|^{n}\left|g_{i}^{\prime}(z)\right|^{n} \leqq \sum_{i=1}^{k}\left|\nabla u\left(g_{i}(z)\right)\right|^{n} K_{O}\left(g_{i}\right) J\left(z, g_{i}\right) \\
& \leqq K_{I}(f) \sum_{i=1}^{k}\left|\nabla u\left(g_{i}(z)\right)\right|^{n} J\left(z, g_{i}\right)
\end{aligned}
$$

Since $K_{I}(f) \leqq K$, this implies

$$
\begin{aligned}
\int_{Q_{j}}|\nabla v|^{n} d m & \leqq K \sum_{i=1}^{k} \int_{Q_{j}}\left|\nabla u\left(g_{i}(z)\right)\right|^{n} J\left(z, g_{i}\right) d m(z) \\
& =K \sum_{i=1}^{k} \int_{g_{i} Q_{j}}|\nabla u|^{n} d m=K \int_{f^{-1} Q_{j}}|\nabla u|^{n} d m .
\end{aligned}
$$


Hence we obtain from (7.18)

$$
\operatorname{cap} f E \leqq K \sum_{j=1}^{\infty} \int_{f^{-1} Q_{j}}|\nabla u|^{n} d m \leqq K \int_{i}|\nabla u|^{n} d m .
$$

Since $u \in W_{0}^{\infty}(E)$ was arbitrary, this proves cap $f E \leqq K$ cap $E$. Theorem 7.1 is thus completely proved.

\section{Applications}

In this section we show that the composite mapping of two quasiregular mappings is quasiregular and that $m\left(B_{f}\right)=0$ for a non-constant quasiregular mapping.

8.1. Theorem. Suppose that $f: G \rightarrow R^{n}$ and $g: G^{\prime} \rightarrow R^{n}$ are quasiregular and that $f G \subset G^{\prime}$. Then $g \circ f: G \rightarrow R^{n}$ is quasiregular, and

$$
K_{o}(g \circ f) \leqq K_{o}(g) K_{o}(f), K_{I}(g \circ f) \leqq K_{I}(g) K_{I}(f) .
$$

Proof. If either $f$ or $g$ is constant, the result is trivial. If not, then $g \circ f$ is sense-preserving, discrete, and open. Let $E$ be a condenser in $G$. Then 7.1 implies

$$
\operatorname{cap} g f E \leqq K_{I}(g) \operatorname{cap} f E \leqq K_{I}(g) K_{I}(f) \operatorname{cap} E .
$$

Hence, by 7.1, $g \circ f$ is quasiregular and $K_{I}(g \circ f) \leqq K_{I}(g) K_{I}(f)$. Since the corresponding result is well known to be valid for quasiconformal mappings, the inequality for $K_{o}$ follows from 2.28 .

8.2. Theorem. If $f: G \rightarrow R^{n}$ is a non-constant quasiregular mapping, then $J(x, f)>0$ a.e.

Proof. We shall use an argument similar to the proof of Theorem 6 in Gehring [9]. It suffices to show that $A=\{x \in G \mid J(x, f)=0\}$ has no points of density.

Let $x_{0} \in G$, and choose a positive number $r_{0}$ such that if $0<r \leqq r_{0}$, then $L^{*}\left(x_{0}, f, r\right) \leqq 2 H^{*}\left(x_{0}, f\right) l^{*}\left(x_{0}, f, r\right)$ and $U\left(x_{0}, f, r\right)$ is a normal neighborhood of $x_{0}$. Fix $r \in\left(0, r_{0}\right]$, and set $U_{0}=U\left(x_{0}, f, r_{0}\right), \quad U=$ $U\left(x_{0}, f, r\right), L^{*}=L^{*}\left(x_{0}, f, r\right)$, and $l^{*}=l^{*}\left(x_{0}, f, r\right)$. Consider the condenser $E=(U, C)$ where $C=\bar{B}^{n}\left(x_{0}, l^{*} / 2\right)$. Let $v \in W_{0}^{\infty}(f E)$, and define $u: U \rightarrow R^{1}$ by $u(x)=v(f(x))$. Since $U$ is a normal domain, spt $u \subset U$. Moreover, $u$ is ACL, and $u(x) \geqq 1$ for $x \in C$. Let $P: R^{n} \rightarrow$ 
$R^{n-1}$ be the orthogonal projection, and set $D=B^{n-1}\left(P\left(x_{0}\right), l^{*} / 2\right)$. Then for almost every $z \in D$ we have

$$
2 \leqq \int_{U \cap P^{-1}(z)}|\nabla u| d m_{1}
$$

Integrating over $z \in D$ yields

$$
\alpha=2^{2-n} \Omega_{n-1} l^{* n-1} \leqq \int_{U}|\nabla u| d m .
$$

Since $|\nabla u(x)| \leqq|\nabla v(f(x))|\left|f^{\prime}(x)\right|$ a.e. and since $f^{\prime}(x)=0$ a.e. in $A$, we obtain

$$
\alpha \leqq \int_{U}|\nabla v(f(x))|\left|f^{\prime}(x)\right| d m(x) .
$$

By Hölder's inequality this implies

$$
\begin{aligned}
& \alpha^{n} \leqq m(U \backslash A)^{n-1} \int_{U}|\nabla v(f(x))|^{n}\left|f^{\prime}(x)\right|^{n} d m(x) \\
\leqq & K_{O}(f) m(U \backslash A)^{n-1} \int_{U}|\nabla v(f(x))|^{n} J(x, f) d m(x) .
\end{aligned}
$$

By [14, Theorem 3, p. 364], this yields

$$
\alpha^{n} \leqq K_{o}(f) N\left(f, U_{0}\right) m(U \backslash A)^{n-1} \int_{f U}|\nabla v|^{n} d m .
$$

Since this holds for all $v \in W_{0}^{\infty}(f E)$, we obtain

$$
\alpha^{n} \leqq K_{o}(f) N\left(f, U_{0}\right) m(U \backslash A)^{n-1} \operatorname{cap} f E .
$$

Here $\operatorname{cap} f E \leqq K_{I}(f) \operatorname{cap} E$ by 7.1 , and $\operatorname{cap} E \leqq \omega_{n-1} /(\log 2)^{n-1}$. Set $B=B^{n}\left(x_{0}, L^{*}\right)$. Then $m(B)=\Omega_{n} L^{* n} \leqq \Omega_{n} 2^{n} H^{*}\left(x_{0}, f\right)^{n} l^{* n}$, and we obtain

$$
m(B) \leqq \beta m(U \backslash A) \leqq \beta m(B \backslash A)
$$

where the constant $\beta$ is independent of $r$. Hence $x_{0}$ cannot be a point of density of $A$.

8.3. Theorem. If $f: G \rightarrow R^{n}$ is a non-constant quasiregular mapping, then $m\left(B_{f}\right)=0$.

Proof. By 2.14 and 2.26, $J(x, f)=0$ a.e. in $B_{f}$. The theorem follows from 8.2. 
8.4. Theorem. Suppose that $f: G \rightarrow R^{n}$ is a non-constant quasiregular mapping. If $A$ is a measurable set in $G$, then $f A$ is measurable. Moreover, $m(f A)=0$ if and only if $m(A)=0$.

Proof. We express $G \backslash B_{f}$ as a countable union of domains in which $f$ is injective. Since $m\left(B_{f}\right)=m\left(f B_{f}\right)=0$, the theorem follows from the corresponding result for quasiconformal mappings.

\section{References}

1. Agard, S.: Angles and quasiconformal mappings in space. - J. Analyse Math. $22(1969), 177-200$.

2. Callender, E. D.: Hölder continuity of $n$-dimensional quasi-conformal mappings. - Pacific J. Math. 10 (1960), 499-515.

3. Černavskir, A. V. (Чернавский, А. В.): Конечнократные открытые отображения многообразий. - Mat. Sbornik 65 (1964), $357-369$.

4. - - - Дополнение к статье "О конечнократных отображениях многообразий". - Mat. Sbornik 66 (1965), 471-472.

5. Church, P. T. and Hemmingsen, E.: Light open maps on $n$-manifolds. - Duke Math. J. 27 (1960), 527-536.

6. Fuglede, B.: Extremal length and functional completion. - Acta Math. 98 (1957), $171-219$.

7. Gehring, F. W.: The definitions and exceptional sets for quasiconformal mappings. - Ann. Acad. Sci. Fenn. A I 281 (1960), 1--28.

8. -》- Symmetrization of rings in space. - Trans. Amer. Math. Soc. 101 (1961), $499-519$.

9. -》- Rings and quasiconformal mappings in space. - Trans. Amer. Math. Soc. 103 (1962), 353-393.

10. - - Extremal length definitions for the conformal capacity of rings in space. . Michigan Math. J. 9 (1962), 137-150.

11. Hocking, J. G. and Young, G. S.: Topology. - Addison-Wesley, 1961.

12. KÜNZI, H. P.: Quasikonforme Abbildungen. - Springer-Verlag, 1960.

13. Lehto, O. and Virtanen, K. I.: Quasikonforme Abbildungen. - Springer-Verlag, 1965.

14. Rado, T. and Reichelderfer, P. V.: Continuous transformations in analysis. Springer-Verlag, 1955.

15. Rеšetnjak, J. G. (Решетняг, Ю. Г.): Оценки моду.ля непрерывности для некоторых отображений. - Sibirsk. Mat. Ž. 7 (1966), $1106-1114$.

16. - -- Пространственные отображения с ограниченным искажением. - Sibirsk. Mat. Ž. 8 (1967), $629-658$.

17. -»- Теорема Лиувилля при минимальных предположенияах регулярности. - Sibirsk. Mat. Ž. 8 (1967), 835-840.

18. -》- Об условии ограниченности индекса для отображений с ограниченным искажением. - Sibirsk. Mat. Ž. 9 (1968), 368-374.

19. -»-- Отображения с ограниченным искажением как экстремали интегралов типа Дирихле. - Sibirsk. Mat. Ž. 9 (1968), 652--666. 
20. -»- Теоремы устойчивости для отображений с ограниченным искажением. - Sibirsk. Mat. Ž. 9 (1968), $667-684$.

21. SAks, S.: Theory of the integral. - Warsaw, 1937.

22. Trtus, C. J. and Young, G. S.: The extension of interiority, with some applications. - Trans. Amer. Math. Soc. 103 (1962), 329-340.

23. VäIsÄLÄ, J.: On quasiconformal mappings in space. - Ann. Acad. Sci. Fenn. A I 298 (1961), $1-36$.

24. -»- Two new characterizations for quasiconformality. - Ann. Acad. Sci. Fenn. A I 362 (1965), 1-12.

25. -»- Discrete open mappings on manifolds. - Ann. Acad. Sci. Fenn. A I 392 (1966), $1-10$.

26. -»- Lectures on $n$-dimensional quasiconformal mappings. - Van Nostrand (to appear).

27. Whyburn, G. T.: Analytic topology. - Amer. Math. Soc. Colloquium Publications, 1942.

28. -»- Open mappings on locally compact spaces. - Mem. Amer. Math. Soc. 1, 1950.

University of Helsinki

Helsinki, Finland 